\title{
A SYSTEMATIC UPSCALING OF NONLINEAR CHEMICAL UPTAKE WITHIN A BIOFILM*
}

\author{
MOHIT P. DALWADI ${ }^{\dagger}$ AND JOHN R. KING ${ }^{\ddagger}$
}

\begin{abstract}
When modeling transport of a chemical species to a colony of bacteria in a biofilm, it is computationally expensive to treat each bacterium even as a point sink, let alone to capture the finite nature of each bacterium. Instead, models tend to treat the bacterial and extracellular matrix domains as a single phase, over which an effective bulk uptake is imposed. In this paper, we systematically derive the effective equations that should govern such a system, starting from the microscale problem of a chemical diffusing through a colony of finite-sized bacteria, within which the chemical species can also diffuse. The uptake within each bacterium is a nonlinear function of the concentration; across the bacterial membrane the concentration flux is conserved and the concentration ratio is constant. We upscale this system using homogenization via the method of multiple scales, investigating the two distinguished limits for the effective uptake and the effective diffusivity, respectively. This work is a natural sequel to Dalwadi et al. [SIAM J. Appl. Math., 78 (2018), 1300-1329], the main difference in this current work being nonlinear uptake within the bacteria and a general partition coefficient across the bacterial membrane. The former results in a significantly more involved general asymptotic analysis, and the latter results in the merging of two previous distinguished limits. We catalogue the different types of microscale behavior that can occur in this system and the effect they have on the observable macroscale uptake. In particular, we show how the nonlinearities in microscale uptake should be modified when upscaled to an effective uptake and how different microscale uptake properties and behaviors, such as chemically depleted regions within the bacteria, can lead to the same observed uptake.
\end{abstract}

Key words. homogenization, multiscale, distinguished limits, effective uptake, quiescent core, depleted core

AMS subject classifications. 35B25, 35B27, 35B40, 92C45

\section{DOI. $10.1137 / 19 \mathrm{M} 130220 \mathrm{X}$}

1. Introduction. The majority of bacteria live communally in biofilms, which can help them survive in hostile environments [11]. This can be a hindrance to humans, since biofilms cause a significant proportion of microbial infections in the body and tend to be extremely resistant to antibiotics $[10,28]$. To understand how to deal with biofilms, it is important to understand how chemicals are absorbed by the bacteria within.

While mathematical modeling can be used to help answer questions involving bacterial chemical uptake, the vast separation of bacterial $(0.1-10 \mu \mathrm{m})$ and biofilm $(0.1-10 \mathrm{~cm})$ lengthscales [16] means that it is prohibitively expensive to include small bacterial regions of uptake in computational models over the lengthscale of a biofilm. A common way to circumvent this issue is by not differentiating between the fluid and bacterial regions in a computational model, treating both regions as a combined single-

*Received by the editors November 25, 2019; accepted for publication (in revised form) April 8, 2020; published electronically July 23, 2020.

https://doi.org/10.1137/19M130220X

Funding: This work was supported by the Biotechnology and Biological Sciences Research Council and the Engineering and Physical Sciences Research Council jointly through the grant BB/L013940/1.

${ }^{\dagger}$ Mathematical Institute, University of Oxford, Radcliffe Observatory Quarter, Oxford, OX2 6GG, UK and Synthetic Biology Research Centre, University of Nottingham, University Park, Nottingham, NG7 2RD, UK (dalwadi@maths.ox.ac.uk).

${ }^{\ddagger}$ School of Mathematical Sciences, University of Nottingham, University Park, Nottingham, NG7 2RD, UK and Synthetic Biology Research Centre, University of Nottingham, University Park, Nottingham, NG7 2RD, UK (john.king@nottingham.ac.uk). 
phase domain and imposing an effective bulk chemical sink over this domain to model the combined effect of many small bacterial regions. While this is certainly a useful mathematical resolution to the problem, it is not always clear how this effective sink should relate to the microscale uptake. For example, how should a nonlinear form of pointwise bacterial uptake transform into an effective bulk uptake? While one might expect the form of uptake to be preserved during an upscaling for weak uptake, the effective results from upscaling are less intuitive when the uptake is stronger. The goal of this paper is to understand and quantify this upscaling procedure in a systematic manner, in terms of the system functions and parameters.

To this end, in this paper we systematically upscale the microscale problem of unsteady chemical diffusion past a locally periodic array of spherical bacteria that act as volumetric sinks with nonlinear kinetics, governed by the reaction-diffusion equation

$$
\frac{\partial C}{\partial t}=\nabla \cdot(\tilde{D} \nabla C)-\tilde{F}(C) .
$$

Here, $\tilde{D}$ is a piecewise-constant function which is discontinuous across each bacterial membrane, and the uptake $\tilde{F}(C) \geqslant 0$ depends on the concentration within each bacterium, but vanishes outside each. We impose a continuous concentration flux across the bacterial membrane, but allow the concentration to be discontinuous across the membrane, coupling the concentration by imposing a constant concentration ratio, also known as a partition coefficient. We provide a schematic of the set-up in Figure 1.

Our main goal in this paper is to determine the effective uptake for the upscaled system when the effective uptake balances diffusion over the biofilm lengthscale, since this yields a balance of transport mechanisms in the upscaled equation. To upscale this problem, we use mathematical homogenization via the method of multiple scales as outlined in, for example, $[2,13,26]$. We derive and investigate the two distinct distinguished limits in the system for the effective uptake and the effective diffusivity, respectively. As different microorganisms will have different microscale parameters, it is helpful to retain the generality provided by the distinguished limit in our analysis.

The work presented here is a natural sequel to [9], in which we considered a subcase of (1) involving a linear uptake function and a unitary partition coefficient, the latter resulting in a continuous concentration across the entire domain. The linear form of the uptake function in [9] meant that we were able to derive analytic expressions for the effective uptake. However, this linearity also meant that the effective uptake was always a linear function (or functional, in Case 2 therein) of the macroscale concentration. For nonlinear uptake, there is no guarantee that the form of the nonlinearity will be preserved during the upscaling procedure, and understanding this is one goal of this paper. In [9] we found that there were three distinguished limits in the system, one for the effective diffusivity (Case 1) and two for the effective uptake (Cases 2 and 3). As one might expect, the inclusion of a general nonlinear uptake in this current work makes the subsequent analysis significantly more involved, and general closed-form solutions are not possible. Instead, noting that it is unlikely that many systems of interest are exactly in the distinguished limit, we systematically consider sublimits of the reduced system we derive for the effective uptake. The presence of a nonunitary partition coefficient means that the two distinguished limits for effective uptake in [9] are actually sublimits of a single distinguished limit for the effective uptake in this work (Case I). To distinguish between the cases in each paper, we use Roman numerals to discuss the two cases we consider in this paper and Arabic numerals to discuss the three cases from [9]. 
There has been a great deal of work in the upscaling of solute transport problems in general, with applications ranging from cell growth in tissue engineering [20], through crystal precipitation/dissolution [30], drug transport in tissue [27], drug delivery to tumors [21], and the electrical activity of cells [23], to solute sorption in soil [22]. Given their ubiquitous nature, significant effort has gone into proving rigorous uniqueness and convergence results for the upscaling of multiscale solute transport problems, e.g., [14].

In each of the papers mentioned in the above paragraph, the structure of the periodic microscale is general within the analysis (although several impose specific microscale structures when performing numerical simulations). This generality is valuable in that it allows homogenized equations to be calculated in terms of general cell problems; however, it also means that effective terms are not derived explicitly.

We consider spherical bacteria (cocci) whose radius can vary slowly over the macroscale, allowing us to model a bacterial density that varies over the lengthscale of the biofilm. While classical homogenization requires a strictly periodic microscale geometry, we use the modern framework that allows one to consider problems with a locally periodic microscale, i.e., a microscale that varies over the macroscale $[3$, $23,30,31]$. The general formulation of this method typically requires a different cell problem to be solved at every point in the macroscale rather than just once for the entire problem, as is the case for classic homogenization. We circumvent this issue by following the examples of $[3,7,8]$ and imposing a specific one-parameter shape on the microstructure, namely, spheres. Moreover, investigating spherical bacteria maximizes the analytic progress we are able to make in determining the effective uptake, yielding greater physical insight into the possible system behaviors.

The structure of this paper is as follows. We present a dimensional description of the bacterial uptake model in section 2 , which we subsequently nondimensionalize. We then formulate the problem to be upscaled via homogenization theory in section 3 . We upscale this problem for the distinguished limit of effective uptake (Case I) in section 4, exploit parameter groupings to reduce the complexity of the steady version of this problem, and present some numerical results of the reduced system. In certain scenarios, the bacteria can exhibit chemical-depleted cores; we investigate the onset of this regime in section 5 . We then investigate various sublimits of the distinguished limit for effective uptake in section 6. Finally, we discuss the implications of our results and conclude in section 7 . For completeness, we also present an upscaling of this problem for the distinguished limit of effective diffusivity (Case II) in Appendix A.

2. Model description. We consider the Fickian diffusion and nonlinear uptake of a chemical species through a colony of bacteria within an extracellular matrix (ECM) which represents a biofilm. Our goal is to systematically upscale the problem over the lengthscale of the bacteria (which we refer to as the microscale) to an effective problem valid over the lengthscale of the bacterial colony (which we refer to as the macroscale) and to investigate what can be deduced about the microscale uptake from macroscale measurements of the effective uptake.

We track the chemical field in terms of its molar concentration, defined in the ECM and bacterial phases as $\tilde{c}$ and $\tilde{C}$, respectively. These concentrations are dependent variables of the independent variables of space $\tilde{\boldsymbol{x}}$ and time $\tilde{\boldsymbol{t}}$. We assume that the chemical species diffuses through the ECM with constant diffusivity $D_{m}$, and through the bacteria with constant diffusivity $D_{b}$. Additionally, we assume that the uptake occurs only within the bacteria, as some general function of the concentration $\tilde{f}(\tilde{C})$. While we aim to consider as general an uptake function as possible, to 
ensure physically relevant uptake we restrict our analysis to systems where there is no uptake when there is no chemical species, i.e., $\tilde{f}(0)=0$, and where an increase in concentration cannot cause a decrease of uptake, i.e., $\tilde{f}$ is a nondecreasing function of $\tilde{C}$.

For simplicity, we model the bacteria as a collection of spheres whose centers are located on a cubic lattice at a distance $\epsilon l$ apart, where $\epsilon$ is a small dimensionless parameter and $l$ is the typical length of the entire domain. To model a bacterial density that can vary in space across the colony, we allow the radii of the bacteria to vary slowly in space. Hence, a bacterium centered at $\tilde{\boldsymbol{x}}$ has radius $\tilde{R}(\tilde{\boldsymbol{x}})$, with the constraint $2 \tilde{R}<\epsilon l$ for nonoverlapping spheres. The bacterial and ECM phases are denoted as $\Omega_{b} \subset \mathbb{R}^{3}$ and $\Omega_{m} \subset \mathbb{R}^{3}$, respectively. We denote the entire spatial domain as $\Omega=\Omega_{b} \cup \Omega_{m} \subset \mathbb{R}^{3}$, noting that $\Omega_{b} \cap \Omega_{m}=\emptyset$. Finally, we denote the boundary between the two phases as $\partial \Omega_{b}$, which we refer to as the "bacterial membrane," or just "membrane." We emphasize that the bacterial phase is disconnected and that the ECM phase is multiply connected.

We assume that the concentrations across the bacterial membrane are coupled through a constant partition coefficient $\mathcal{K}$ and through continuity of concentration flux. We assume that the concentration field is initially known and consistent with the coupling conditions discussed above and that any (initial) spatial variations occur over the lengthscale of the colony rather than that of the bacteria. The setup we describe here is similar to that in [9], but now with a nonlinear uptake and a general partition coefficient instead of a linear uptake and unitary partition coefficient, respectively. We will show that the former change causes markedly different macroscale behavior compared to [9], while the latter causes different distinguished asymptotic limits.

Mathematically, we have the dimensional problem

$$
\begin{array}{r}
\frac{\partial \tilde{c}}{\partial \tilde{t}}=D_{m} \nabla^{2} \tilde{c} \quad \text { for } \tilde{\boldsymbol{x}} \in \Omega_{m}, \quad \frac{\partial \tilde{C}}{\partial \tilde{t}}=D_{b} \nabla^{2} \tilde{C}-\tilde{f}(\tilde{C}) \quad \text { for } \tilde{\boldsymbol{x}} \in \Omega_{b}, \\
\tilde{c}=\mathcal{K} \tilde{C}, \quad \boldsymbol{n} \cdot D_{m} \nabla \tilde{c}=\boldsymbol{n} \cdot D_{b} \nabla \tilde{C} \quad \text { for } \tilde{\boldsymbol{x}} \in \partial \Omega_{b}, \\
\tilde{c}(\tilde{\boldsymbol{x}}, 0)=\tilde{c}_{\text {init }}(\tilde{\boldsymbol{x}}) \quad \text { for } \tilde{\boldsymbol{x}} \in \Omega_{m}, \quad \tilde{C}(\tilde{\boldsymbol{x}}, 0)=\tilde{c}_{\text {init }}(\tilde{\boldsymbol{x}}) / \mathcal{K} \quad \text { for } \tilde{\boldsymbol{x}} \in \Omega_{b},
\end{array}
$$

where $\boldsymbol{n}$ is the unit normal of the bacterial membrane pointing into the surrounding ECM. The function $\tilde{c}_{\text {init }}(\tilde{\boldsymbol{x}})$ appearing in the initial conditions $(2 \mathrm{c})$ is continuous across the bacterial membrane and allows for a slow variation of the chemical concentration in space. To close the system (2), we also require boundary conditions at the external boundary of $\Omega$. However, to keep the generality of our analysis we will not impose a specific form in this paper.

It is helpful to discuss parameter values for the problem we have introduced. Spherical bacteria, known as cocci, have a typical diameter of around $2 \tilde{R} \approx 1 \mu \mathrm{m}$, and an approximate cell spacing of $\epsilon l \approx 2-20 \mu \mathrm{m}[9,16,17]$. It is possible to obtain the diffusion coefficient of a given chemical within water; for example, the diffusivity of dissolved oxygen within water is approximately $2 \times 10^{-9} \mathrm{~m}^{2} \mathrm{~s}^{-1}$ at room temperature. In a similar manner, the partition coefficients for many different chemicals across an octanol/water interface is well-known, and these can vary across many orders of magnitude [15]. However, it is significantly more difficult to obtain diffusivity, partition coefficient, and uptake measurements involving cytoplasm, partly due to the difficulties in isolating and imaging a single bacterium. For this reason, and in order to present a more general analysis of the problem, it will be instructive to consider the various distinguished asymptotic limits of this problem. 


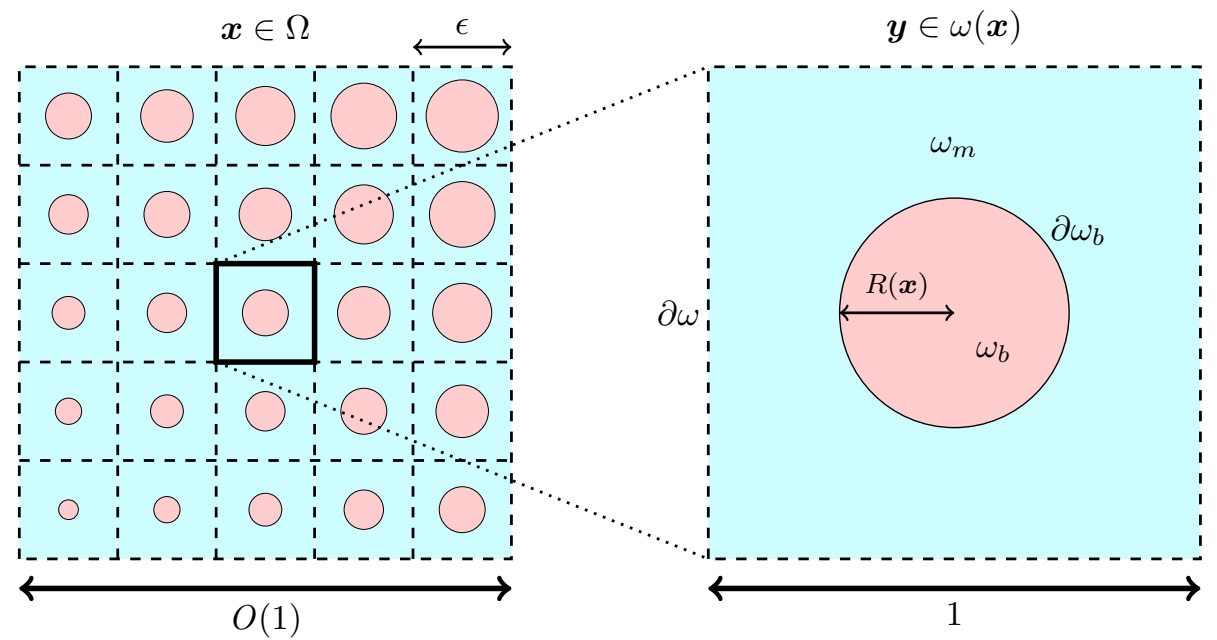

FIG. 1. A two-dimensional projection of the three-dimensional problem. The full problem is shown in the left-hand figure, and the cell problem (with $\boldsymbol{y} \in[-1 / 2,1 / 2]^{3}$ ) is shown in the right-hand figure. The pink regions denote the bacteria and the blue region denotes the ECM.

2.1. Dimensionless equations. We scale the variables via $\tilde{\boldsymbol{x}}=l \boldsymbol{x}, \tilde{t}=\left(l^{2} / D_{m}\right) t$, $\tilde{R}=\epsilon l R,\left(\tilde{c}, \tilde{C}, \tilde{c}_{\text {init }}\right)=c_{\infty}\left(c, C, c_{\text {init }}\right)$, and $\tilde{f}(\tilde{C})=\left(c_{\infty} D_{m} / l^{2}\right) f(C)$, where $c_{\infty}$ is a characteristic concentration scale in the ECM, to yield the dimensionless equations

$$
\begin{aligned}
& \frac{\partial c}{\partial t}=\nabla^{2} c \quad \text { for } \boldsymbol{x} \in \Omega_{m}, \quad \frac{\partial C}{\partial t}=D \nabla^{2} C-f(C) \quad \text { for } \boldsymbol{x} \in \Omega_{b}, \\
& c=\mathcal{K} C \\
& \boldsymbol{n} \cdot \nabla c=\boldsymbol{n} \cdot D \nabla C \\
& \text { for } \boldsymbol{x} \in \partial \Omega_{b} \text {, } \\
& \text { for } \boldsymbol{x} \in \partial \Omega_{b} \text {, } \\
& c(\boldsymbol{x}, 0)=c_{\text {init }}(\boldsymbol{x}) \quad \text { for } \boldsymbol{x} \in \Omega_{m}, \quad C(\boldsymbol{x}, 0)=c_{\text {init }}(\boldsymbol{x}) / \mathcal{K} \quad \text { for } \boldsymbol{x} \in \Omega_{b},
\end{aligned}
$$

where $D=D_{b} / D_{m}$ is the ratio of chemical diffusivity in the ECM to that in the bacteria. We do not specify the asymptotic orders of these dimensionless parameters yet, but our interest will be in the distinguished asymptotic limits where the timescale of effective uptake balances the timescale of macroscale diffusion in the ECM, in which $t=O(1)$.

In dimensionless units, the bacteria now form a cubic lattice of spheres whose centers are a distance of $\epsilon$ apart, and a bacterium centered at $\boldsymbol{x}$ has radius $\epsilon R(\boldsymbol{x})$. A schematic of this set-up is shown in Figure 1.

3. Deriving effective equations. Our goal is to upscale the governing equations (3) using a homogenization procedure via the method of multiple scales. Essentially, we introduce the additional spatial variable

$$
\boldsymbol{y}=\frac{\boldsymbol{x}-\lfloor\boldsymbol{x}\rfloor}{\epsilon}-\boldsymbol{b},
$$

where we treat $\boldsymbol{x}$ and $\boldsymbol{y}$ as independent. In (4), we introduce the constant translation vector $\boldsymbol{b}=(1 / 2,1 / 2,1 / 2)$ for notational purposes. Thus, the microscale variable $\boldsymbol{y} \in[-1 / 2,1 / 2]^{3}$ is defined within a unit cell $\omega(\boldsymbol{x})$, centered around one bacterium, and our dependent variables are now $c(\boldsymbol{x}, \boldsymbol{y}, t)$ and $C(\boldsymbol{x}, \boldsymbol{y}, t)$. As is standard in homogenization via the method of multiple scales, the extra freedom that arises from 
introducing $\boldsymbol{y}$ is later removed by imposing that the problem is 1-periodic in each component of $\boldsymbol{y}$.

Within each cell, we define several regions for convenience. The bacterium and ECM phases are defined as $\omega_{b}(\boldsymbol{x})$ and $\omega_{m}(\boldsymbol{x})$, respectively. The spherical bacterial membrane between these two phases is defined as $\partial \omega_{b}(\boldsymbol{x})$. Finally, the cubic outer boundary of the cell is defined as $\partial \omega$. These regions are all labeled in Figure 1.

We are interested in deriving effective governing equations for the experimentally measurable concentration $\widehat{c}(\boldsymbol{x}, t)$, the intrinsic-averaged concentration within the ECM, defined as

$$
\widehat{c}(\boldsymbol{x}, t)=\frac{1}{\left|\omega_{m}(\boldsymbol{x})\right|} \int_{\omega_{m}(\boldsymbol{x})} c(\boldsymbol{x}, \boldsymbol{y}, t) \mathrm{d} \boldsymbol{y},
$$

where $\left|\omega_{m}\right|$ is the volume of the ECM phase in one cell.

Treating each dependent variable as a function of both $\boldsymbol{x}$ and $\boldsymbol{y}$, the spatial derivatives transform as follows:

$$
\nabla \mapsto \nabla_{\boldsymbol{x}}+\frac{1}{\epsilon} \nabla_{\boldsymbol{y}}
$$

where $\nabla_{\boldsymbol{x}}$ and $\nabla_{\boldsymbol{y}}$ refer to the nabla operator in the $\boldsymbol{x}$ - and $\boldsymbol{y}$-coordinate systems, respectively. The spatial transformation (6) also causes the unit normal on the boundary to transform (as also occurs in, for example, $[3,30]$ ). This can be seen by defining the function $\chi(\boldsymbol{x}, \boldsymbol{y})=\|\boldsymbol{y}\|-R(\boldsymbol{x})$, noting that the bacterial membrane is defined by $\chi=0$ and thus $\boldsymbol{n}=\nabla \chi /\|\nabla \chi\|$, then using (6) to yield

$$
\boldsymbol{n} \mapsto \frac{\boldsymbol{n}_{\boldsymbol{y}}-\epsilon \nabla_{\boldsymbol{x}} R}{\left\|\boldsymbol{n}_{\boldsymbol{y}}-\epsilon \nabla_{\boldsymbol{x}} R\right\|},
$$

where $\boldsymbol{n}_{\boldsymbol{y}}=\boldsymbol{y} /\|\boldsymbol{y}\|$. This transformation is also known as the level-set framework [31]. come

$$
\left(\boldsymbol{n}_{\boldsymbol{y}}-\epsilon \nabla_{\boldsymbol{x}} R\right) \cdot\left(\nabla_{\boldsymbol{y}}+\epsilon \nabla_{\boldsymbol{x}}\right) c=\left(\boldsymbol{n}_{\boldsymbol{y}}-\epsilon \nabla_{\boldsymbol{x}} R\right) \cdot D\left(\nabla_{\boldsymbol{y}}+\epsilon \nabla_{\boldsymbol{x}}\right) C \quad \text { for } \boldsymbol{y} \in \partial \omega_{b}(\boldsymbol{x}),
$$

$$
\begin{aligned}
c(\boldsymbol{x}, \boldsymbol{y}, 0)=c_{\text {init }}(\boldsymbol{x}) \quad \text { for } \boldsymbol{y} \in \omega_{m}(\boldsymbol{x}), \quad C(\boldsymbol{x}, \boldsymbol{y}, 0)=c_{\text {init }}(\boldsymbol{x}) / \mathcal{K} & \text { for } \boldsymbol{y} \in \omega_{b}(\boldsymbol{x}), \\
c \text { periodic } & \text { for } \boldsymbol{y} \in \partial \omega,
\end{aligned}
$$

where (8f) is required to remove secular terms, as is standard in the method of multiple scales.

We are interested in the physical scenarios in which the effective uptake balances the macroscale diffusion over the timescale of the latter, $t=O(1)$. We summarize two different balances which yield a distinguished asymptotic limit in the macroscale problem in Table 1. In order to get a sense of from where these asymptotic limits 
TABLE 1

The scalings for the two distinguished asymptotic limits. Case I is the distinguished limit for the effective uptake, and Case II is the distinguished limit for the effective diffusivity. Note that $\left|\omega_{b}\right| \sim R^{3}$ has already been scaled by $\epsilon^{3}$ so that it is of the same asymptotic order as the periodic-cell size when $\left|\omega_{b}\right|=O(1)$.

\begin{tabular}{c|ccccc}
\multicolumn{1}{c}{} & $D$ & $f$ & $\left|\omega_{b}\right|$ & $\mathcal{K}$ & $C$ \\
\cline { 2 - 6 } Case I & $O\left(\epsilon^{6}\right)$ & $O\left(1 / \epsilon^{6}\right)$ & $O\left(\epsilon^{6}\right)$ & $O\left(\epsilon^{6}\right)$ & $O\left(1 / \epsilon^{6}\right)$ \\
Case II & $O(1)$ & $O(1)$ & $O(1)$ & $O(1)$ & $O(1)$
\end{tabular}

arise, it is helpful to use (3) to consider the removal rate of the total chemical species in the system, in the absence of any source/sink at the external boundary

$$
\frac{\partial}{\partial t}\left(\int_{\omega_{m}} c \mathrm{~d} \boldsymbol{x}+\int_{\omega_{b}} C \mathrm{~d} \boldsymbol{x}\right)=-\int_{\omega_{b}} f(C) \mathrm{d} \boldsymbol{x} .
$$

When bacterial uptake occurs over the entire bacterium and not just within a boundary layer near the bacterial membrane, we see from (9) that an uptake timescale of $t=O(1)$ occurs when $f(C)\left|\omega_{b}\right|=O(1)$, noting that $\left|\omega_{m}\right|=O(1)$ and $c=O(1)$ (the latter being true away from any boundary layer near an external boundary). Additionally, the coupling conditions (8c)-(8d) also yield $\mathcal{K} C=O(1)$ and $D C=O(1)$. The remaining scalings arise through seeking a balance between diffusion and uptake at leading-order within each bacterium, as will be seen through our analysis.

4. Case I: Distinguished limit for the effective uptake. The distinguished limit for the effective uptake occurs when the timescale of microscale uptake balances that of macroscale diffusion, retaining as many terms from the governing equation (8) at leading-order as possible. Preserving the timescale of macroscale diffusion, this distinguished limit occurs when the bacteria are very sparse $(R \ll 1)$, the pointwise uptake is very strong $f(C) \gg 1$, the chemical species has a strong preference for the bacterial cytoplasm $(\mathcal{K} \ll 1)$, and the chemical diffusivity within the cytoplasm is very small $(D \ll 1)$. With appropriate scalings that we discuss below, we are able to retain all terms at leading-order within the bacterial domain. Investigating this distinguished limit will allow us to derive the effective uptake in its most general form. We can then explore relevant sublimits to obtain physical insight into this system. This distinguished limit for the effective uptake contains Cases 2 and 3 from [9] as asymptotic sublimits.

4.1. Asymptotic structure. The formal scalings we consider are $C=\bar{C} / \epsilon^{6}$, $D=\epsilon^{6} \bar{D}, R=\epsilon^{2} \bar{R}, f(C)=\bar{f}(\bar{C}) / \epsilon^{6}$, and $\mathcal{K}=\epsilon^{6} \overline{\mathcal{K}}$, where the new inner variables are labeled with an overline and are each of $O(1)$. These scalings ensure that the leadingorder inner problem within the bacterium retains all terms at leading-order. Note that we have already scaled $R$ with the microscale variable, so in terms of dimensionless macroscale variables we are considering the case where the radius scales with the cube of the small parameter of periodicity, the critical case in $[6,18]$.

In this section, our analysis involves upscaling the governing equations (3) using a combination of boundary layer analysis and homogenization via the method of multiple scales. There are three important asymptotic regions in this problem. The first is the outer region, $\boldsymbol{x}=O(1)$. This is the lengthscale over which we want to determine an effective equation which systematically accounts for the bacterial uptake. Thus in the outer region the bacterial uptake appears as a bulk effect from our homogenization procedure. The second (intermediate) region is the cell region, $\boldsymbol{x}=O(\epsilon)$. This region will yield the cell problem and, in this region, the bacterial 


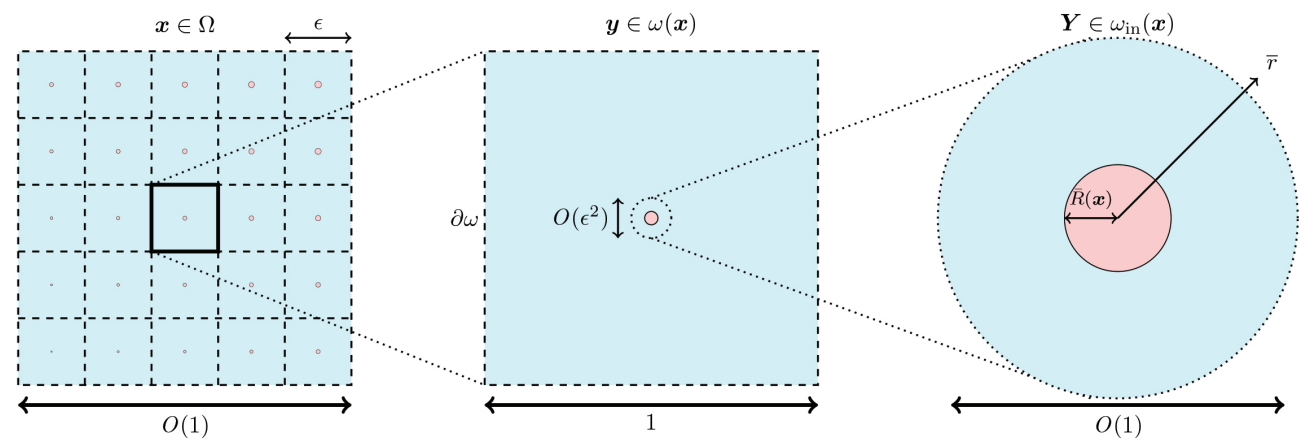

FIG. 2. A two-dimensional projection of the asymptotic structure of the three-dimensional problem, involving the distinguished limit for the effective uptake. The full problem in the outer region is shown in the left figure, the center figure denotes the cell region (with $\boldsymbol{y} \in[-1 / 2,1 / 2]^{3}$ ), and the rightmost figure denotes the inner region within the cell region (with $\boldsymbol{Y} \in \mathbb{R}^{3}$ and $\bar{r}=\|\boldsymbol{Y}\|$ ). In the cell region, the effect of the bacterial sink appears as a delta function, and not through the problem geometry. The strength of this sink is determined by solving the problem in the inner region.

uptake appears as a point sink. The third and final region is the inner region, $\boldsymbol{x}=$ $O\left(\epsilon^{3}\right)$. In this region, the finite nature of a bacterium is apparent, and it appears as a region of $O(1)$ volume surrounded by ECM, within which we must solve a coupled concentration problem. The solution from the inner region will determine the strength of the point sink in the cell region, which will determine the effective uptake over the outer region. A schematic of these three regions is given in Figure 2.

4.2. Homogenization. Using the scalings discussed above, we rewrite the equations (8) in the cell region as follows:

$$
\begin{array}{r}
\left(\boldsymbol{n}_{\boldsymbol{y}}-\epsilon^{3} \nabla_{\boldsymbol{x}} \bar{R}\right) \cdot\left(\nabla_{\boldsymbol{y}}+\epsilon \nabla_{\boldsymbol{x}}\right) c=\left(\boldsymbol{n}_{\boldsymbol{y}}-\epsilon^{3} \nabla_{\boldsymbol{x}} \bar{R}\right) \cdot \bar{D}\left(\nabla_{\boldsymbol{y}}+\epsilon \nabla_{\boldsymbol{x}}\right) \bar{C} \text { for }\|\boldsymbol{y}\|=\epsilon^{2} \bar{R}, \\
(10 \mathrm{e}) \\
(10 \mathrm{x}) \\
(10 \mathrm{~g}) \\
\bar{C}(\boldsymbol{x}, 0)=c_{\text {init }}(\boldsymbol{x}) \text { for }\|\boldsymbol{y}\|>\epsilon^{2} \bar{R} \text { and }\|\boldsymbol{y}\|_{\infty}<1 / 2, \\
c \text { init }(\boldsymbol{x}) / \overline{\mathcal{K}} \text { for }\|\boldsymbol{y}\|<\epsilon^{2} \bar{R}, \\
c \text { periodic for }\|\boldsymbol{y}\|_{\infty}=1 / 2,
\end{array}
$$

where $\|\cdot\|$ is the Euclidean norm and $\|\cdot\|_{\infty}$ is the maximum norm. We cannot obtain a solution for $\bar{C}$ by simply expanding in powers of $\epsilon$, since the bacterial domain in (10) depends on the small parameter $\epsilon$. Instead, we seek an inner solution to the system about a (small) bacterium at the origin, in which $\|\boldsymbol{y}\|=O\left(\epsilon^{2}\right)$. In the next section, we show that the inner solution only affects the governing equation for $c$ in the cell region at $O\left(\epsilon^{2}\right)$. Thus, substituting the asymptotic expansion $c(\boldsymbol{x}, \boldsymbol{y}, t) \sim c_{0}(\boldsymbol{x}, \boldsymbol{y}, t)+$ $\epsilon c_{1}(\boldsymbol{x}, \boldsymbol{y}, t)+\epsilon^{2} c_{2}(\boldsymbol{x}, \boldsymbol{y}, t)$ into (10a) implies that $c_{0}=c_{0}(\boldsymbol{x}, t)$ and $c_{1}=c_{1}(\boldsymbol{x}, t)$. Therefore, the average concentration (5) behaves as $\widehat{c} \sim c_{0}$. We now investigate the inner region. 
4.3. Inner region. To move into this region we scale $\boldsymbol{y}=\epsilon^{2} \boldsymbol{Y}$, where $\boldsymbol{Y} \in \mathbb{R}^{3}$. We define this inner region as $\omega_{\text {in }}(\boldsymbol{x})$, where the dependence on $\boldsymbol{x}$ arises from the radius of the bacterium in this domain. From (10), the relevant leading-order system is

$$
\begin{aligned}
\nabla_{\boldsymbol{Y}}^{2} c=0 & \text { for } \bar{r}>\bar{R}(\boldsymbol{x}), \\
\frac{\partial \bar{C}}{\partial t}=\bar{D} \nabla_{\boldsymbol{Y}}^{2} \bar{C}-\bar{f}(\bar{C}) & \text { for } 0<\bar{r}<\bar{R}(\boldsymbol{x}), \\
c=\overline{\mathcal{K}} \bar{C} \quad \frac{\partial c}{\partial \bar{r}}=\bar{D} \frac{\partial \bar{C}}{\partial \bar{r}} & \text { for } \bar{r}=\bar{R}(\boldsymbol{x}), \\
c \rightarrow c_{0}(\boldsymbol{x}, t) \sim \widehat{c}(\boldsymbol{x}, t) & \text { as } \bar{r} \rightarrow \infty, \\
\bar{C}=c_{\text {init }}(\boldsymbol{x}) / \overline{\mathcal{K}} & \text { for } \bar{r}<\bar{R}(\boldsymbol{x}) \text { at } t=0,
\end{aligned}
$$

where $\bar{r}=\|\boldsymbol{Y}\|$. The far-field condition (11d) arises from matching with the cell region using van Dyke's matching principle [29].

We now reduce the system (11) to a closed system for $\bar{C}$ in terms of the effective concentration $\widehat{c}$ and the microscale parameters. This is possible since the bacteria are spherical, so (11a) and (11d) imply

$$
c=c_{0}(1-B / \bar{r})
$$

for some function $B(\boldsymbol{x}, t)$. Substituting the solution (12) into the interfacial conditions (11c), we obtain

$$
\bar{C}=\frac{c_{0}}{\overline{\mathcal{K}}}\left(1-\frac{B(\boldsymbol{x}, t)}{\bar{R}}\right), \quad \bar{D} \frac{\partial \bar{C}}{\partial \bar{r}}=\frac{c_{0} B(\boldsymbol{x}, t)}{\bar{R}^{2}} \quad \text { for } \bar{r}=\bar{R}(\boldsymbol{x}) .
$$

We are able to remove $B$ from (13) by combining both equations, allowing us to derive the following Robin boundary condition for $\bar{C}$,

$$
\overline{\mathcal{K}} \bar{C}+\bar{R} \bar{D} \frac{\partial \bar{C}}{\partial \bar{r}}=\widehat{c}(\boldsymbol{x}, t) \quad \text { for } \bar{r}=\bar{R}(\boldsymbol{x}),
$$

and we note this also tells us that $B=\left(\widehat{c}-\left.\overline{\mathcal{K}} \bar{C}\right|_{\bar{r}=\bar{R}}\right) / \bar{R}$. Thus, we have derived a reduced problem which consists of solving a single nonlinear parabolic equation in a finite domain (11b), with a Robin boundary condition on the bacterial membrane (14a), and a symmetry condition at the bacterium center

$$
\frac{\partial \bar{C}}{\partial \bar{r}}=0 \quad \text { for } \bar{r}=0 .
$$

Solving the closed inner system (11b), (14) will allow us to determine the effective uptake over the macroscale domain. We show in section 4.4 that this is equal to the total flux in the far-field, so it is helpful to define the functional

$$
\nu[\widehat{c}]:=4 \pi \lim _{\bar{r} \rightarrow \infty} \bar{r}^{2} \frac{\partial c}{\partial \bar{r}}=\left.4 \pi \bar{R}^{2} \bar{D} \frac{\partial \bar{C}}{\partial \bar{r}}\right|_{\bar{r}=\bar{R}},
$$

which, we shall show later, defines the effective uptake in the upscaled problem. Given the form of $\nu$ and the boundary condition (14a), we are able to make the following general statement about $\nu$, no matter the specific details of the pointwise nonlinear uptake function. Given that $\bar{C}$ and $\partial \bar{C} / \partial \bar{r}$ are both nonnegative, (14a) implies that 
$0 \leqslant \bar{D} \partial \bar{C} / \partial \bar{r} \leqslant \widehat{c} / \bar{R}$ at the cell membrane. Hence, from (15) we may obtain the bounds

$$
0 \leqslant \nu[\widehat{c}] \leqslant 4 \pi \bar{R} \widehat{c} .
$$

Since the closed problem we have derived in this section represents the distinguished limit for the effective uptake, we will return to it later to investigate its steady states further, after we understand how this problem relates to the effective uptake over the macroscale.

4.4. Higher-order cell region problem. Given the far-field form of the concentration field in the inner problem (12), we must introduce a Dirac delta function into the cell region problem (10) at $O\left(\epsilon^{2}\right)$ to match appropriately. This represents the effect of the bacterial uptake region over the cell region. From this, the $O\left(\epsilon^{2}\right)$ terms from (10) are

$$
\begin{aligned}
\frac{\partial \widehat{c}}{\partial t}=\nabla_{\boldsymbol{y}}^{2} c_{2}+\nabla_{\boldsymbol{x}}^{2} \widehat{c}-\hat{\delta}(\boldsymbol{y}) \nu[\widehat{c}] & \text { for } \boldsymbol{y} \in \omega, \\
c_{2} \text { periodic } & \text { for } \boldsymbol{y} \in \partial \omega,
\end{aligned}
$$

where we have used $\widehat{c} \sim c_{0}$.

Integrating (17a) over the periodic cell and applying the periodic boundary conditions (17b), we obtain the effective equation for the intrinsic-averaged concentration

$$
\frac{\partial \widehat{c}}{\partial t}=\nabla_{\boldsymbol{x}}^{2} \widehat{c}-\nu[\widehat{c}],
$$

where $\nu$ is defined in (15) and is derived by solving (11b) with boundary conditions (14). The initial condition for this system is

$$
\widehat{c}(\boldsymbol{x}, 0)=c_{\text {init }}(\boldsymbol{x}),
$$

which arises from substituting (10e) into (5). We note that the effective uptake here is of $O(1)$, and hence the macroscale uptake balances macroscale diffusion.

The effective diffusivity in Case I is one, corresponding to that of pure ECM and representing the fact that chemical diffusivity within the cytoplasm is unimportant when the bacteria are very small. A nontrivial effective diffusivity arises in Case II, which we present in Appendix A.

To give an idea of the form of the effective uptake $\nu[\widehat{c}]$, we give the solution to the inner problem (11b) with boundary conditions (14) for a linear uptake, $\bar{f}(\bar{C})=\bar{\mu} \bar{C}$. The analysis is similar to that of Cases 2 and 3 in [9], where we solely consider linear uptake, in which reduced versions of this inner problem are derived. In this case, $\bar{C}$ can be solved for as follows,

$$
\bar{C}=\frac{\widehat{c}}{\overline{\mathcal{K}}}-\frac{2}{\bar{R} \bar{D} \bar{r}} \sum_{n=1}^{\infty} \frac{\sin \lambda_{n} \bar{R} \sin \lambda_{n} \bar{r}}{\lambda_{n}^{2}} e^{-\left(\bar{\mu}+\bar{D} \lambda_{n}^{2}\right) t} \int_{0}^{t}\left(\frac{\partial \widehat{c}}{\partial \tau}+\bar{\mu} \widehat{c}\right) e^{\left(\bar{\mu}+\bar{D} \lambda_{n}^{2}\right) \tau} \mathrm{d} \tau
$$

where the eigenvalues are the countably infinite positive solutions to the following transcendental equation,

$$
(\overline{\mathcal{K}}-\bar{D}) \sin \lambda_{n} \bar{R}+\bar{D} \lambda_{n} \bar{R} \cos \lambda_{n} \bar{R}=0 .
$$


From (19), we may deduce the following form for the effective uptake:

$$
\nu[\widehat{c}]=\frac{8 \pi \overline{\mathcal{K}}}{\bar{D} \bar{R}} \sum_{n=1}^{\infty} \frac{\sin ^{2} \lambda_{n} \bar{R}}{\lambda_{n}^{2}} e^{-\left(\bar{\mu}+\bar{D} \lambda_{n}^{2}\right) t} \int_{0}^{t}\left(\frac{\partial \widehat{c}}{\partial \tau}+\bar{\mu} \widehat{c}\right) e^{\left(\bar{\mu}+\bar{D} \lambda_{n}^{2}\right) \tau} \mathrm{d} \tau .
$$

We see from (20) that the system history is important for the functional $\nu$. Given the integral in (20), we expect this memory property to have a smoothing influence for $\nu$.

As the problem we have presented in section 4 represents the distinguished limit for the effective uptake, it will be instructive to investigate the inner problem and how it affects the effective uptake in more detail. The analysis of its steady state is the remaining focus of this paper, so it is helpful to present the reduced steady problem.

4.5. System reduction. To simplify our analysis of how the effective uptake depends on the inner problem, we consider the steady problem. Since all the steady states of this system are stable (Appendix B) and we expect the effective uptake to smooth out its history, the steady state is of fundamental interest.

The steady-state version of the inner problem (11b), (14) is

$$
\begin{array}{r}
0=\frac{\bar{D}}{\bar{r}^{2}} \frac{\partial}{\partial \bar{r}}\left(\bar{r}^{2} \frac{\partial \bar{C}}{\partial \bar{r}}\right)-\bar{f}(\bar{C}) \quad \text { for } 0<\bar{r}<\bar{R}(\boldsymbol{x}), \\
\frac{\partial \bar{C}}{\partial \bar{r}}=0 \quad \text { for } \bar{r}=0, \quad \overline{\mathcal{K}} \bar{C}+\bar{R} \bar{D} \frac{\partial \bar{C}}{\partial \bar{r}}=\widehat{c}(\boldsymbol{x}) \quad \text { for } \bar{r}=\bar{R}(\boldsymbol{x}),
\end{array}
$$

and the effective uptake over the macroscale is the total concentration flux into this system (15). As the macroscale parameter $\boldsymbol{x}$ only appears as a parameter in this inner problem, for notational purposes we henceforth suppress any functional dependence on $\boldsymbol{x}$.

We are able to scale out several of the parameters in the system (21) by introducing $\bar{r}=\bar{R} r, \bar{C}=(\widehat{c} / \bar{D}) w$, and $\bar{f}(\bar{C})=\widehat{c} F(w) / \bar{R}^{2}$ to transform (21) into

$$
\begin{gathered}
0=\frac{1}{r^{2}} \frac{\partial}{\partial r}\left(r^{2} \frac{\partial w}{\partial r}\right)-F(w) \quad \text { for } 0<r<1, \\
\frac{\partial w}{\partial r}=0 \quad \text { for } r=0, \quad k w+\frac{\partial w}{\partial r}=1 \quad \text { for } r=1,
\end{gathered}
$$

where $k=\overline{\mathcal{K}} / \bar{D}$. Under this scaling the effective uptake (15) becomes

$$
\nu[\widehat{c}]=\left.4 \pi \bar{R} \widehat{c} \frac{\partial w}{\partial r}\right|_{r=1} .
$$

Restricting the problem to the steady state means the effective uptake reduces from a functional to a function of $\widehat{c}$. However, since $w$ may have a nonlinear dependence on $\widehat{c}$ through the scalings of $w$ and $F$, the effective uptake will still have a nonlinear dependence on $\widehat{c}$ in general.

To contextualize our asymptotic results in the following sections, it is helpful to understand how our scaling transforms a power-law uptake. Such an uptake can arise via, e.g., the law of mass action [19], with homopolymerization. For example, if $\widehat{c}$ were a monomer that had to be dimerized before uptake, we could have a power-law uptake with exponent 2. Alternatively, if $\widehat{c}$ were a dimer that had to be dissociated before uptake, we could have a power-law uptake with exponent 1/2. A power-law uptake is transformed as follows:

$$
\bar{f}(\bar{C})=\bar{\mu} \bar{C}^{\beta} \quad \mapsto \quad F(w)=\mu w^{\beta} \quad \text { with } \mu=\frac{\bar{\mu} \bar{R}^{2} \widehat{c}^{\beta-1}}{\bar{D}^{\beta}} .
$$



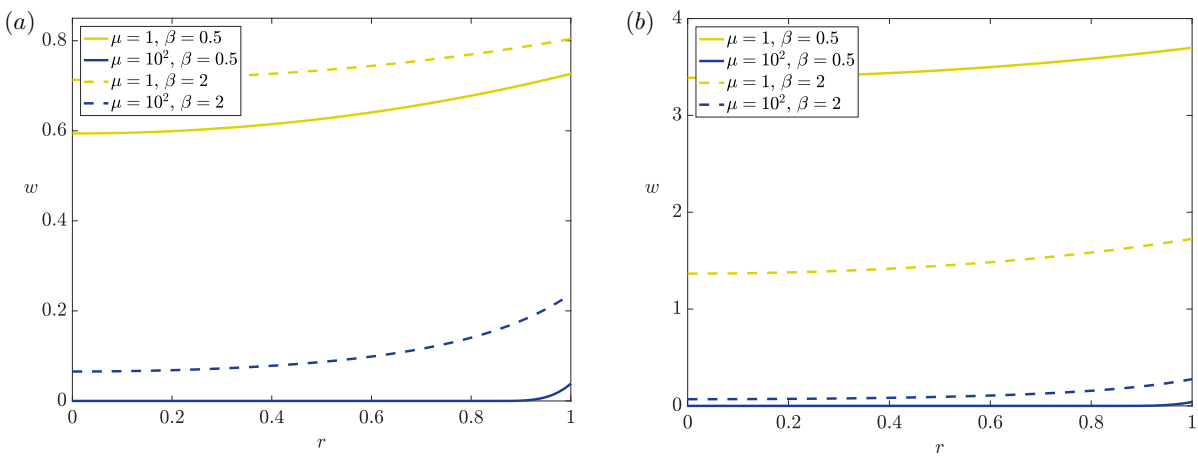

FIG. 3. Solutions to the system (22) using a power-law uptake of the form $F(w)=\mu w^{\beta}$ with (a) $k=1$ and (b) $k=0.1$.

In particular, we note that large $\widehat{c}$ corresponds to large $\mu$ if $\beta>1$ and to small $\mu$ if $\beta<1$, and vice versa for small $\widehat{c}$.

4.5.1. Numerical results. We can solve the system (22) in MATLAB for given uptake function $F(w)$ and parameter $k$, using the in-built boundary-value solver bvp5c, which essentially iterates an initial guess for the solution (we use $w=1 / k$ ) until the numerical solution falls below a specified relative error tolerance $\left(10^{-3}\right.$ in our simulations). As one might expect, a larger $\mu$ corresponds to a smaller $w$ (Figure 3). However, in terms of the power-law uptake given in (24), the relationship between the size of $w$ and $\beta$ is nonmonotonic. In addition, the uptake is non-Lipschitz when $\beta<1$, and this can result in a situation where the bacterial core is depleted of the chemical species, as shown in the $\mu=10^{2}, \beta=0.5$ cases in Figure 3. Despite the fundamental change in the nature of the solution at the onset of core depletion, we are still able to approximate the solution numerically using the method outlined above, since we iterate to within a specified error tolerance. However, we note that we would need to take significantly more care with our numerical method if we wanted to determine the position of the free boundary. We explore the onset of the free-boundary regime in more detail in section 5 .

The important quantity from this analysis is $\partial w / \partial r$ evaluated at $r=1$, which is fundamentally related to the effective uptake over the entire colony of bacteria from (23). As one may expect, the effective uptake increases as $\mu$ increases (Figure 4). However, this increase has severe diminishing returns since $\partial w / \partial r$ is bounded above by 1 , as predicted by (16). Since $\partial w / \partial r$ evaluated at $r=1$ appears to vary smoothly as the parameters in the system vary smoothly, the onset of a concentration-depleted bacterial core for certain parameter values does not have a significant effect on the effective uptake, despite the drastic change in the microscale problem.

To understand these observations in more detail, we investigate the system analytically in the remainder of the paper, interrogating various sublimits of the problem. We start by investigating the onset of the depleted bacterial core.

5. Depleted core. As noted in section 4.5.1, there exist solutions in which the bacteria contain a concentration-depleted core. In this section, we characterize the onset of this behavior for a power-law uptake with the form $F(w)=\mu w^{\beta}$ for $0<\beta<1$ in the system (22). That is, in this section we solely consider uptake functions with a non-Lipschitz power-law form. After the onset of the core-depletion regime, the 
(a)

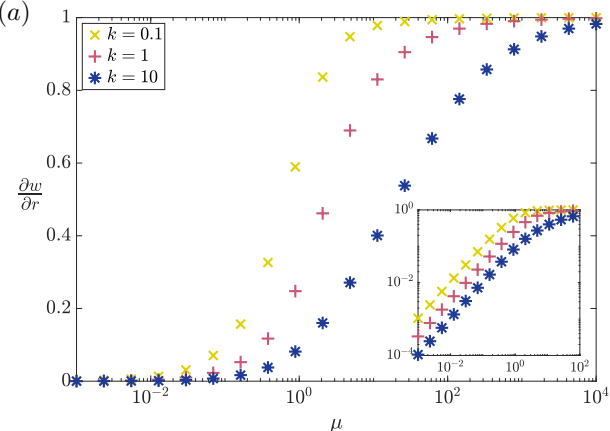

(b)

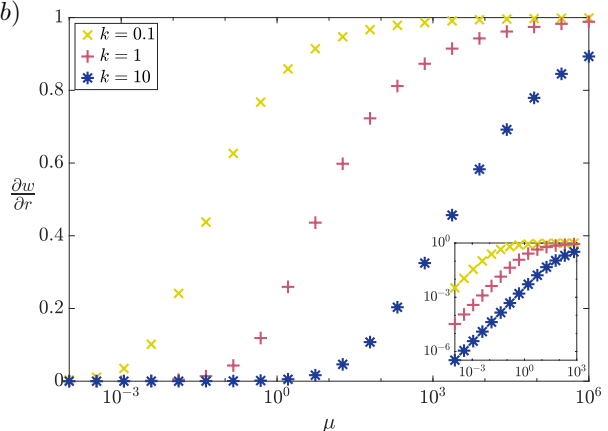

FIG. 4. The derivative of the solution to the system (22), evaluated at the boundary $r=1$, which feeds into the effective uptake over the entire system, as defined in (23). We use a power-law uptake of the form $F(w)=\mu w^{\beta}$ with (a) $\beta=0.5$ and (b) $\beta=2$.

system becomes a free-boundary problem. That is, depletion means that (22) is only valid in $r_{c}<r<1$ with $w=0$ for $0<r<r_{c}$. At the free boundary $r=r_{c}$, we may close the problem and prescribe $r_{c}$ by imposing continuity of concentration and concentration flux, i.e., $w\left(r_{c}\right)=0$ and $w^{\prime}\left(r_{c}\right)=0$. As this regime represents a fundamental change in the solution structure, it is helpful to be able to calculate the point at which this occurs; the onset of this regime is the subject of this section.

There is a critical surface in $(\beta, k, \mu)$ parameter space that separates the original fixed-boundary problem and the free-boundary problem outlined above. This critical surface occurs at the onset of the depletion regime, which corresponds to $r_{c}=0$. At this point, (22) can be solved using the power-law solution

$$
w=\left(\frac{\mu(1-\beta)^{2} r^{2}}{2(3-\beta)}\right)^{\frac{1}{1-\beta}} \quad \text { for } 0<r<1 \text {, }
$$

which defines the following critical surface in $(\beta, k, \mu)$ parameter space:

$$
\mu\left(k+\frac{2}{1-\beta}\right)^{1-\beta}=\frac{2(3-\beta)}{(1-\beta)^{2}} .
$$

From the scalings in (24), we see that $\mu$ has an inherent $\beta$-dependence. Hence, we use the prescaled variables in section 4.5 (essentially replacing $k$ with $\overline{\mathcal{K}} / \bar{D}$ and $\mu$ with $\left.\bar{\mu} \bar{R}^{2} \widehat{c}^{\beta-1} / \bar{D}^{\beta}\right)$ to illustrate the onset of the depletion regime in Figure 5 . We see that increasing $\bar{\mu} \bar{R}^{2}$ has the effect of increasing the domain of parameter space in which depletion can occur. Moreover, there is a critical value of $\bar{\mu} \bar{R}^{2}$ above which the critical line becomes nonmonotonic in $(\widehat{c}, \beta)$-space. Additionally, we note that the critical value of $\widehat{c}$ becomes very small as $\beta \rightarrow 1^{-}$.

To quantify these observations, we calculate the following asymptotic results (in terms of the prescaled variables in section 4.5) for the onset of the depletion regime:

$$
\begin{gathered}
\widehat{c} \sim \frac{\bar{\mu} \bar{R}^{2}(\overline{\mathcal{K}}+2 \bar{D})}{6 \bar{D}}\left[1+\beta\left(\log \frac{\mu \bar{R}^{2}}{6 \bar{D}}-\frac{5 \overline{\mathcal{K}}+4 \bar{D}}{3(\overline{\mathcal{K}}+2 \bar{D})}\right)\right] \quad \text { as } \beta \rightarrow 0^{+}, \\
\widehat{c} \sim \frac{2 \bar{D} \sqrt{e}}{1-\beta}\left(\frac{\bar{\mu} \bar{R}^{2}(1-\beta)^{2}}{4 \bar{D}}\right)^{\frac{1}{1-\beta}} \quad \text { as } \beta \rightarrow 1^{-} .
\end{gathered}
$$

Copyright (C) by SIAM. Unauthorized reproduction of this article is prohibited. 


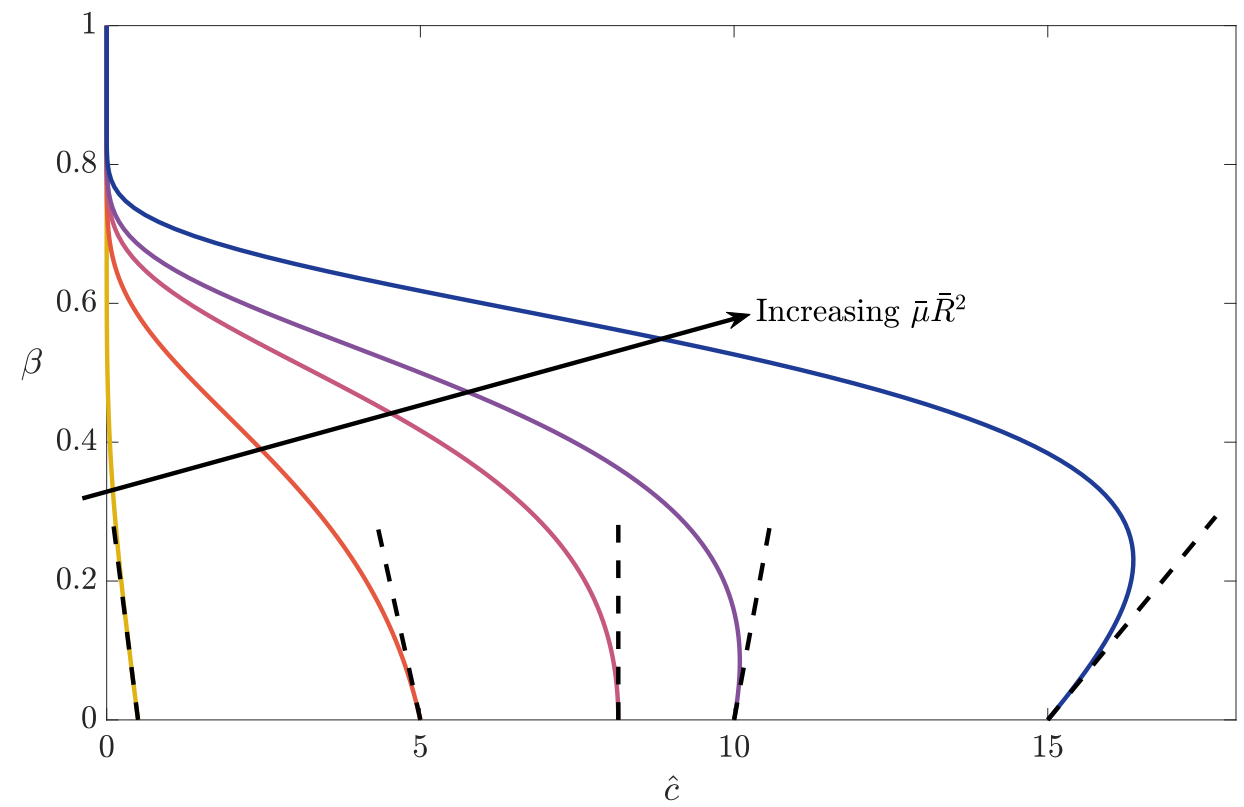

FIG. 5. The critical lines defining the onset of depletion for different uptake strength, calculated from the nonlinear algebraic equation (26). Depletion occurs to the left of each critical line. We transform $\mu$ into $\bar{\mu}$ using (24) and use $\overline{\mathcal{K}}=1, \bar{D}=1$ throughout. In terms of these parameters, the critical value where the extent of the depletion zone starts to increase as $\beta$ is increased is $\bar{\mu} \bar{R}^{2}=6 e \approx 16.31$, from (28). We use values of $\bar{\mu} \bar{R}^{2} \in\{1,10,6 e, 20,30\}$. The dashed black lines denote the small $\beta$ results (27a).

From (27a), we see that there is a critical value of $\bar{\mu}=\bar{\mu}^{*}$, where $\partial \widehat{c} / \partial \beta=0$ on the critical line at $\beta=0$. Across this critical value, the critical line switches from being monotonic in parameter space to being nonmonotonic, as seen in Figure 5. This critical value is given by

$$
\bar{\mu}^{*}=\frac{6 \bar{D}}{\bar{R}^{2}} \exp \left(\frac{5 \overline{\mathcal{K}}+4 \bar{D}}{3(\overline{\mathcal{K}}+2 \bar{D})}\right) .
$$

While there is an exponential dependence in (28), the value of $\bar{\mu}^{*}$ is not especially sensitive to the parameter values in the system. This is because the argument of the exponential in (28) can only take values within $(2 / 3,5 / 3)$, so the exponential term can only vary by a factor of $e$. For the parameter values used in Figure $5, \bar{\mu}^{*}=6 e \approx 16.31$. With regard to our observation above regarding the extent of the depletion regime as $\beta \rightarrow 1^{-}$, we may deduce from (27b) that the depletion regime does exist for all $0<\beta<1$, but that its extent becomes exponentially small as $\beta \rightarrow 1^{-}$.

6. Asymptotic limits of Case I. In this section we investigate various asymptotic limits of the system (22) and deduce how these limits affect the effective uptake. We then discuss how these results apply to different physical limits in the system. We discuss the three main cases of fast $(F \gg w)$, moderate $(F=O(w))$, and slow $(F \ll w)$ uptake separately, as we will show that they each exhibit distinct behavior. We name these cases in reference to the speed of their uptake timescales compared to the timescale of diffusive transport across a single bacterium, in dimensional terms. In each case, we identify and use the asymptotic size of $k$ that gives a distinguished limit for that case, in order to keep our analysis as general as possible. However, 
we also consider sublimits of $k$ where instructive. As opposed to the power-law form assumed in section 5, our analysis in this section applies to general nonlinear uptake functions $F(w)$, though we will discuss how our results apply to power-law uptakes where relevant.

6.1. Fast uptake: $\boldsymbol{F} \gg \boldsymbol{w}$. In the case of fast uptake, there is a boundary layer near the membrane within the bacterium, through which the concentration rapidly decreases, and therefore $w$ must be small to allow the derivative in the boundary condition $(22 \mathrm{~b})$ to balance one of the additional terms. We have a full distinguished limit if $k \gg 1$, and we show the reasoning for this below. Performing this scaling analysis systematically, we may derive the scalings $r=1-\delta \rho, w=\delta W$, and $F(w)=$ $G(W) / \delta$, where $\delta$ satisfies the implicit equation

$$
\delta F(\delta)=1 .
$$

Since $F(z) \gg z$ here, we emphasize that $\delta \ll 1$. We also scale $k=\kappa / \delta$ to obtain a distinguished limit, and the reason for this scaling will be made apparent below. In our new boundary layer coordinate, $\rho=0$ corresponds to the bacterial membrane and $\rho>0$ corresponds to the bacterial domain.

From the scalings presented above, we obtain the leading-order system

$$
\begin{aligned}
0=\frac{\partial^{2} W}{\partial \rho^{2}}-G(W) & \text { for } \rho>0, \\
\kappa W-\frac{\partial W}{\partial \rho}=1 & \text { for } \rho=0, \\
W \rightarrow 0 & \text { for } \rho \rightarrow \infty .
\end{aligned}
$$

We obtain the last condition via asymptotic matching, noting that $W$ will become asymptotically smaller away from the interface in order to obtain a balance between uptake and diffusion in the bulk bacterial domain.

We can in the usual way solve (30) implicitly, by first multiplying (30a) by $\partial W / \partial \rho$ and integrating with respect to $\rho$ :

$$
\left(\frac{\partial W}{\partial \rho}\right)^{2}=2 \int_{0}^{W} G(s) \mathrm{d} s
$$

where we have invoked (30c) (and additionally $\partial W / \partial \rho \rightarrow 0$ as $\rho \rightarrow \infty$ ). For later use, we note that combining $(30 \mathrm{~b})$ and (31) leads to the following implicit representation of $W(0)$ :

$$
(1-\kappa W(0))^{2}=2 \int_{0}^{W(0)} G(s) \mathrm{d} s .
$$

Since the left-hand side of (32) monotonically decreases from 1 to 0 as $W(0)$ increases from 0 to $1 / \kappa$, and the right-hand side monotonically increases from a value of 0 to a positive value as $W(0)$ increases from 0 to $1 / \kappa,(32)$ has a unique solution $W(0) \in$ $(0,1 / \kappa)$.

As $W$ decreases when we move into the bacterial domain, we may rewrite (31) as

$$
\frac{\partial W}{\partial \rho}=-\sqrt{2 \int_{0}^{W} G(s) \mathrm{d} s},
$$

Copyright (C) by SIAM. Unauthorized reproduction of this article is prohibited. 


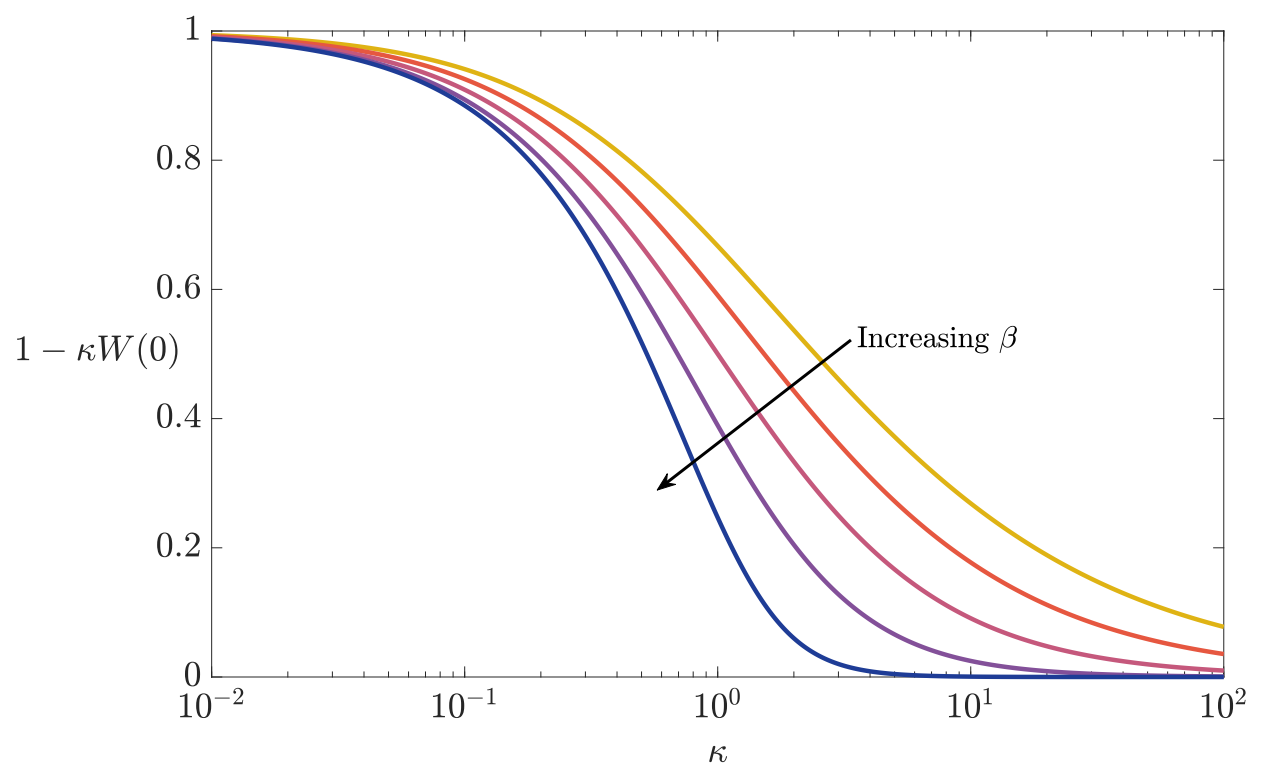

FIG. 6. The solution to the nonlinear equation (39) for a power-law uptake. We plot $1-\kappa W(0)$, which is related to the effective uptake through (36). We use values of $\beta \in\{0.2,0.5,1,2,5\}$, noting that $W(0)=1 /(1+\kappa)$ for $\beta=1$.

which we can integrate to obtain the following implicit representation for $W$,

$$
\int_{W}^{W(0)} \frac{\mathrm{d} u}{\sqrt{2 \int_{0}^{u} G(s) \mathrm{d} s}}=\rho,
$$

recalling that $W(0)$ is defined in (32).

The implicit solution (34) is not necessarily valid through the entire boundary layer if the uptake function is non-Lipschitz. In such scenarios, the representation (34) is valid only for $0<\rho<\rho^{*}$ with $W \equiv 0$ for $\rho \geqslant \rho^{*}$, resulting in a chemically depleted core. We are able to calculate this critical point in closed form as follows:

$$
\rho^{*}:=\int_{0}^{W(0)} \frac{\mathrm{d} u}{\sqrt{2 \int_{0}^{u} G(s) \mathrm{d} s}} .
$$

Although we can use the implicit representation (34) to obtain the effective uptake (23), it is simpler to use the leading-order boundary condition (30b) to deduce that, in the superlinear case,

$$
\nu[\widehat{c}] \sim 4 \pi \bar{R}(1-\kappa W(0)) \widehat{c},
$$

where $W(0) \in(0,1 / \kappa)$ is the unique solution to (32). Hence, in the fast-uptake case we have reduced the problem of determining the effective uptake to the problem of solving a nonlinear algebraic equation (32) in terms of the nonlinear uptake function $G$. We show $\nu / 4 \pi \bar{R} \widehat{c}=1-\kappa W(0)$ for a power-law uptake in Figure 6. 
There are some further reductions of complexity possible for asymptotic limits of the scaled partition coefficient $\kappa$ in the nonlinear equation (32). In particular,

$$
\nu[\widehat{c}] \sim 4 \pi \bar{R} \widehat{c} \quad \text { if } \kappa \rightarrow 0^{+}, \quad \nu[\widehat{c}] \sim\left(4 \pi \bar{R} \sqrt{2 \int_{0}^{1 / \kappa} G(s) \mathrm{d} s}\right) \widehat{c} \quad \text { if } \kappa \rightarrow \infty,
$$

and we note that the effective uptake decreases as $\kappa$ increases. In the small- $\kappa$ limit, the effective uptake is bounded above and independent of all parameters in the system except the bacterial radius and external concentration. Moreover, in the same limit the effective uptake is insensitive to the form of the nonlinear pointwise uptake. This insensitivity in the observable effective uptake belies the radically different behavior possible on the microscale; it is striking that the macroscale behavior can be the same even when there are fundamental differences in the microscale behavior such as, for example, the presence or absence of a chemically depleted core within a bacterium.

In the specific case where the uptake satisfies a power law, with $F(w)=\mu w^{\beta}$ for $\beta>0$, fast uptake occurs when $\mu \gg 1$. In this case, we see from (29) that $\delta=\mu^{-\frac{1}{\beta+1}}$, and the implicit solution (34) can be made explicit:

$$
W=\left((W(0))^{\frac{1-\beta}{2}}+\frac{(\beta-1) \rho}{\sqrt{2(\beta+1)}}\right)^{\frac{2}{1-\beta}} .
$$

From (32), $W(0)$ satisfies the nonlinear equation

$$
(1-\kappa W(0))^{2}=\frac{2(W(0))^{\beta+1}}{\beta+1} \text { for } W(0) \in(0,1 / \kappa) .
$$

When $0<\beta<1$, the solution (38) is only valid for $0<\rho<\rho^{*}$, with critical depletion point

$$
\rho^{*}=\sqrt{\frac{2(\beta+1)(W(0))^{\beta+1}}{(1-\beta)^{2}}},
$$

and, as stated previously, $W \equiv 0$ for for $\rho>\rho^{*}$ when depletion occurs.

In terms of the effective uptake (36), we note that $\kappa$ is scaled with $\delta$, which is a function of $\widehat{c}$. Therefore, the effective uptake will scale with $\widehat{c}$ in a manner that must be determined by solving (39). However, we can use our general asymptotic results (37) for extreme values of $\kappa$ to note the following results in terms of the prescaled parameters in section 4.5 ,

$$
\begin{aligned}
& \nu[\widehat{c}] \sim 4 \pi \bar{R} \widehat{c} \quad \text { if } \frac{\overline{\mathcal{K}}^{\beta+1}}{\bar{\mu} \overline{\bar{D}} \bar{R}^{2} \widehat{c}^{\beta-1}} \ll 1, \\
& \nu[\widehat{c}] \sim 4 \pi \bar{R}^{2} \sqrt{\frac{2 \bar{\mu} \bar{D}}{(\beta+1) \overline{\mathcal{K}}^{\beta+1}}} \widehat{c}^{\frac{\beta+1}{2}} \quad \text { if } \frac{\overline{\mathcal{K}}^{\beta+1}}{\bar{\mu} \bar{D} \bar{R}^{2} \widehat{c}^{\beta-1}} \gg 1,
\end{aligned}
$$

and we note that the separate requirement of fast uptake, $\mu \gg 1$, corresponds to $\bar{\mu} \bar{R}^{2} \widehat{c}^{\beta-1} / \bar{D}^{\beta} \gg 1$. We see from (41) that the effective uptake can scale from anywhere between the bacterial radius to the bacterial surface area. Additionally, we note that a pointwise power-law uptake with exponent $\beta$ can result in an effective uptake that scales as $\widehat{c}^{(\beta+1) / 2}$ in the large- $\overline{\mathcal{K}}$ limit and can scale linearly with the effective 
concentration in the small- $\overline{\mathcal{K}}$ limit. Therefore, we can infer that observing a power-law dependence on the concentration in the effective uptake does not mean the pointwise uptake satisfies the same power-law form, even for a linear effective uptake. If the order of magnitude of physical bacterial parameters is known, our results can be used to infer details of the microscale uptake, given observations of the effective uptake for a varying concentration.

6.2. Moderate uptake: $\boldsymbol{F}=\boldsymbol{O}(\boldsymbol{w})$. For a balanced uptake, there is a distinguished limit when $k=O(1)$. In this scenario, the full system is (22), so we cannot make generic analytic progress. In the asymptotic limit $k \ll 1$, the boundary condition at the cell membrane (22b) reduces to a Neumann condition. In this case, even though we cannot obtain a general reduced solution for $w$, we are able to use this simplification of (22b) to immediately deduce that the effective uptake (23) becomes

$$
\nu[\widehat{c}] \sim 4 \pi \bar{R} \widehat{c} \quad \text { as } k \rightarrow 0^{+} .
$$

In the asymptotic limit of $k \gg 1,(22 \mathrm{~b})$ reduces to the Dirichlet condition $k w=1$ with $w=O(1 / k)$. If $k F(1 / k)=O(1)$ in this sublimit, we would still have to solve the remaining system numerically to determine the effective uptake. If not, we could use the results we provide for fast (section 6.1) or slow (section 6.3) uptake.

For completeness, we also present the steady results for the linear case where $F(w)=\mu w$. While we give a representation of the solution and effective uptake in (19)-(20) for the full time-dependent problem, the steady solution can be written in terms of analytic functions, avoiding the need for an infinite sum. In the steady linear case, (21) is solved by

$$
w=\frac{\sinh \sqrt{\mu} r}{r((k-1) \sinh \sqrt{\mu}+\sqrt{\mu} \cosh \sqrt{\mu})},
$$

which is similar to the equivalent result obtained in [9], but now including the effect of the scaled partition coefficient $k$. The corresponding effective uptake (23) is

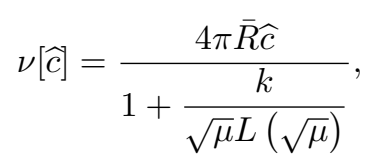

where $L$ is the Langevin function, defined as

$$
L(z)=\operatorname{coth} z-\frac{1}{z}
$$

We can therefore deduce that if the pointwise uptake is linear in the intrinsic concentration, the effective uptake will be linear in the effective concentration. In terms of the prescaled parameters in section 4.5, we note that $F(w)=\mu w$ corresponds to $\bar{f}(\bar{C})=\bar{\mu} \bar{C}$ with $\mu=\bar{\mu} \bar{R}^{2} / \bar{D}$. As the strength of the linear pointwise uptake becomes large and $\bar{\mu} \rightarrow \infty$, the effective uptake in (44) scales as $\nu \sim 4 \pi \bar{R} \widehat{c}$ and carries no information about the underlying microscale uptake. We also note that as $\bar{\mu} \rightarrow 0^{+}$, the effective uptake in (44) scales as $\nu \sim\left|\omega_{b}\right| \bar{\mu} \widehat{c} / \mathcal{K}$, scaling with bacterial volume.

6.3. Slow uptake: $\boldsymbol{F} \ll \boldsymbol{w}$. In the case of slow uptake within the bacteria, the asymptotic solution to (22) is a constant plus a small correction to account for this slow uptake. From this, we pose the following asymptotic ansatz for $w$ :

$$
w(r)=A+F(A) W_{1}(r)+O\left(F(A) F^{\prime}(A)\right),
$$


where $A$ is a constant to be determined. There is a full distinguished limit if $A=$ $O(1 / k) \gg 1$ with $F(1 / k)=O(1)$, and we show the reasoning for this below. Substituting (46) into (22), the leading-order system implies that $A$ is indeed constant. The value of this constant can be determined by closing the system at the next order, which is

$$
\begin{gathered}
0=\frac{1}{r^{2}} \frac{\partial}{\partial r}\left(r^{2} \frac{\partial W_{1}}{\partial r}\right)-1 \quad \text { for } 0<r<1, \\
\frac{\partial W_{1}}{\partial r}=0 \quad \text { for } r=0, \quad k A+\frac{\partial W_{1}}{\partial r} F(A)=1 \quad \text { for } r=1 .
\end{gathered}
$$

The second condition in (47b) shows why we require the balances $A=O(1 / k) \gg 1$ with $F(1 / k)=O(1)$ for a distinguished limit. We can solve (47) for $W_{1}$ up to a constant $B$, as follows:

$$
W_{1}=\frac{1}{6}\left(r^{2}-B\right)
$$

The boundary condition at the bacterial membrane (47b) then yields the following nonlinear equation for $A$ :

$$
k A+\frac{F(A)}{3}=1
$$

We note that (49) could alternatively be derived by invoking the divergence theorem on the full inner problem (22), in conjunction with the ansatz (46). As $A$ and $F(A)$ are both monotonic increasing functions of $A$, there is exactly one solution to (49) with $A>0$. Using the results (46) and (48) to determine the effective uptake (23), we obtain

$$
\nu[\widehat{c}]=\frac{4}{3} \pi \bar{R} \widehat{c} F(A) .
$$

We have therefore reduced the problem of determining the effective uptake to the problem of solving the nonlinear algebraic equation (49) for a given uptake function $F$. We are also able to reduce the complexity further in various limits. First, if $k \gg F(A) / A$, then $A \sim 1 / k$, and the effective uptake can simply be read off from (50). Second, if $k \ll F(A) / A$ and $F(s)>3$ as $s \rightarrow \infty$, then (49) asymptotically reduces to $A=F^{-1}(3)$. That is, as the pointwise uptake increases, the effective uptake behaves as $\nu[\widehat{c}] \sim 4 \pi \bar{R} \widehat{c}$ and conveys no information about the microscale uptake to the macroscale.

Although we do not need to determine $B$ in order to obtain results about the effective uptake, it can be obtained by going to $O\left(F(A) F^{\prime}(A)\right)$ in the asymptotic expansion (46). For completeness, but omitting the details for brevity, we thereby obtain

$$
B=\frac{3}{5} \frac{F^{\prime}(A)+5 k}{F^{\prime}(A)+3 k} \in(0.6,1) .
$$

In terms of the prescaled parameters of section 4.5, the effective uptake (23) is

$$
\nu[\widehat{c}]=\left|\omega_{b}\right| \bar{f}(\widehat{c} A / \bar{D}),
$$

where $A$ satisfies the nonlinear equation (49), which can also be rewritten as

$$
\frac{\overline{\mathcal{K}}}{\bar{D}} A+\frac{\bar{R}^{2} \bar{f}(\widehat{c} A / \bar{D})}{3 \widehat{c}}=1
$$


Since $\bar{f}(z) \ll z$, in order for both terms on the right-hand side of $(52 \mathrm{~b})$ to contribute to $A$ we require $3 \overline{\mathcal{K}} / \bar{R}^{2}=O(\bar{f}(\widehat{c} A / \bar{D}) /(\widehat{c} A / \bar{D}))$. If this is not the case, we may obtain the following asymptotic results for the effective uptake:

$$
\begin{array}{r}
\nu[\widehat{c}] \sim\left|\omega_{b}\right| \bar{f}(\widehat{c} / \overline{\mathcal{K}}) \quad \text { if } \overline{\mathcal{K}} \gg \frac{\bar{R}^{2}}{3} \bar{f}(\widehat{c} / \overline{\mathcal{K}}), \\
\nu[\widehat{c}] \sim 4 \pi \bar{R} \widehat{c} \quad \text { if } \widehat{c} \ll \frac{\bar{R}^{2}}{3} \bar{f}(\widehat{c} / \overline{\mathcal{K}}) .
\end{array}
$$

Hence, the effective uptake in this case can scale from bacterial volume to radius, and from a nonlinear function of the concentration that mirrors the pointwise uptake to a linear function of concentration which carries no information about the underlying microscale uptake.

7. Discussion. We systematically upscale the microscale problem of a chemical species diffusing through an extracellular matrix containing a locally periodic array of spherical bacteria, modeling a biofilm. The chemical can diffuse within the bacteria, which also act as volume sinks of the chemical species, with general nonlinear kinetics. Across the cell membrane, the concentration ratio is a prescribed constant and the concentration flux is conserved. Using homogenization via the method of multiple scales, we derive effective reaction-diffusion equations over the macroscale that systematically account for the microscale bacterial details. Through an asymptotic analysis, we investigate the distinguished limits for the effective uptake in section 4 (Case I) and the effective diffusivity in Appendix A (Case II), in each case where the effective uptake balances the macroscale diffusion over the timescale of the latter. In the key sublimits of fast and slow uptake within the bacteria, we reduce the problem of determining the effective uptake (which generally involves solving a nonlinear PDE) to that of solving a nonlinear algebraic equation for each case. These results represent the effective uptake and diffusivity that should be imposed in biofilm models involving chemical uptake, as they have been systematically derived in terms of microscale bacterial properties.

The distinguished limit for the effective uptake is a double-porosity model [1] in the sense that it occurs when the diffusivity varies greatly between the inside and the outside of the bacteria, by a factor that depends on the separation of scales in the problem. Upscaling this double-porosity model results in two coupled partial differential equations, one for the macroscale diffusion which includes a term that accounts for the microscale uptake, the latter being determined by solving a coupled problem on the microscale. However, as this distinguished limit involves several different microscale parameters, there are many physically relevant sublimits where these macroscale equations will decouple. As such, in section 6 we investigate in detail the steady version of the reduced system that governs the effective uptake, in an effort to catalogue the different types of behavior that this system can exhibit. We show that the effective uptake over the macroscale, the lengthscale over which uptake is measured, can have a very different form from the actual nonlinear pointwise uptake occurring within the bacteria.

In terms of dimensional quantities, the steady effective equations we derive in Case I (for a distinguished limit of effective uptake) have the following form:

$$
D_{m} \nabla^{2} \tilde{c}=\tilde{\nu}[\tilde{c}],
$$

where $\tilde{\nu}$ is the dimensional version of the effective uptake we calculate in various asymptotic limits, and we define the system parameters in section 2. Fast and slow 
uptake (investigated in sections 6.1 and 6.3, respectively) correspond to the timescale of uptake compared to the timescale of diffusion across the bacteria, i.e., $\tilde{R}^{2} / D_{b} \gg$ $\tilde{C} / \tilde{f}(\tilde{C})$ and $\tilde{R}^{2} / D_{b} \ll \tilde{C} / \tilde{f}(\tilde{C})$, respectively. For fast uptake, we deduce that

$$
\tilde{\nu}[\tilde{c}] \sim \frac{4 \pi \tilde{R}^{2}}{(\epsilon l)^{3}} \sqrt{2 D_{b} \int_{0}^{\zeta[\tilde{c}]} \tilde{f}(s) \mathrm{d} s}
$$

where $\zeta[\tilde{c}]$ satisfies the following nonlinear equation:

$$
(\tilde{c}-\mathcal{K} \zeta)^{2}=\frac{2 \tilde{R}^{2} D_{b}}{D_{m}^{2}} \int_{0}^{\zeta} \tilde{f}(s) \mathrm{d} s
$$

For slow uptake, we deduce that

$$
\tilde{\nu}[\tilde{c}] \sim \frac{4 \pi \tilde{R}^{3}}{3(\epsilon l)^{3}} \tilde{f}(\eta[\tilde{c}]),
$$

where $\eta[\tilde{c}]$ satisfies the following nonlinear equation:

$$
\tilde{f}(\eta[\tilde{c}])+\frac{3 D_{m} \mathcal{K}}{\tilde{R}^{2}} \eta[\tilde{c}]=\frac{3 D_{m} \tilde{c}}{\tilde{R}^{2}} .
$$

As can be deduced from (55)-(56), for a strong pointwise uptake the observed uptake appears as a linear function of concentration, even though the microscale behavior is very different in each limit. In this case, the effective uptake is bounded above and cannot be improved by increasing the pointwise uptake through, for example, a genetic modification to up-regulate enzymes associated with an uptake of the chemical of interest. Moreover, (55)-(56) show that a nonlinear observed uptake may have a significantly different nonlinear form compared to the actual pointwise uptake, e.g., a different exponent in a power-law uptake.

As our upscaling procedure results in an effective reaction-diffusion equation, nontrivial steady solutions are possible for suitable boundary conditions. Moreover, since the coefficients of this effective equation can depend on the spatial coordinate, a wide range of interesting steady-state behaviors are possible. For example, in the limiting behavior given in (41b), it is the square root of the microscale uptake coefficient which is important at the macroscale. This demonstrates that a very wide class of effective uptake functions are possible at the macroscale, depending on what type of heterogeneity is present at the microscale.

The results we derive in this paper provide a cautionary tale for anyone attempting to infer microscale kinetics occurring within bacteria from macroscale observations or trying to use experimental results of enzyme kinetics measured in a cell-free environment to model bacterial uptake in, for example, biofilms. In general, when modeling the chemical uptake over a colony of bacteria, the mathematical form of the uptake requires careful thought. In this paper, we systematically derive the correct uptake to impose in terms of the bacterial properties and the measured results from cell-free enzyme assays. Moreover, our results show that the inverse problem of understanding the microscale uptake from macroscale observations is ill-posed in general. For example, there are several different types of microscale behavior that lead to an observed effective uptake which is linear with chemical concentration. The microscale problem could involve chemically depleted zones for fast non-Lipschitz pointwise uptake, as shown in sections 5 and 6.1 ; the partition coefficient could be much smaller than the 
ratio of bacterial-to-ECM diffusivity for slow uptake, as shown in section 6.3 , or the pointwise uptake could simply be inherently linear, as shown in section 6.2.

In both Cases I and II, the leading-order concentration within the ECM does not depend on the microscale variable over the lengthscale of bacterial separation, except very close to the bacteria in Case I. This means that our results are not restricted to bacteria arranged in a cubic lattice. In fact, due to their leading-order independence of the microscale, our effective uptake results will formally hold for any Bravais lattice of spheres, with an appropriate factor to account for the relative phase volumes. Moreover, since the distinguished limit for effective uptake does not include any information about neighboring bacteria, we also expect our effective uptake results to hold for randomly placed bacteria, again with an appropriate factor to account for the relative phase volumes. However, we expect the effective diffusivity we calculate to strongly depend on the microscale structure, as the geometry of the cell problem will change. It would be interesting to formally investigate this problem with stochastically placed bacteria, using the framework of $[4,5,24,25]$, for example.

The difference in microscale dependence of the effective diffusivity and uptake can be understood by considering the classical homogenization of a one-dimensional reaction-diffusion equation (see, for example, [12, section 5.2]), where the effective diffusivity is determined to be the harmonic average of the periodic diffusivity and the effective uptake is determined to be the arithmetic average of the periodic uptake. The harmonic average causes the effective diffusivity to be very sensitive to low diffusivity regions. In higher dimensions, this also causes a sensitivity on the relative positions of such regions, as low diffusivity trapping regions will have a greater effect on effective diffusivity. The same is not true for the effective uptake, which remains as an arithmetic average of the (weak) pointwise uptake for higher dimensions. Thus the effective uptake in the classical case only depends on the microscale structure through an appropriate factor to account for the relative phase volumes. We note that our work in Case II can be applied directly to more general periodic arrays of arbitrary bacterial shapes; the relevant effective diffusion coefficients can be obtained from (68c) by solving the cell problem (64) for different geometries. However, although the general reduced inner problem we derive in (11) does hold for arbitrary bacterial shapes, the reduced boundary condition (14a) does not: this is because we require spherical bacteria to derive (14a), exploiting the separability of Laplace's equation in spherical coordinates. As such, we expect that further analytic progress could be made for bacterial shapes defined by a surface in other separable coordinate systems, such as ellipsoidal bacteria, as considered in the appendix of [9].

A more generalized version of the partition coupling condition across the bacterial membrane $(3 \mathrm{~b})$ is

$$
\Pi(c-\mathcal{K} C)=\boldsymbol{n} \cdot \nabla c
$$

for constant permeability $\Pi$. The condition (57) can be formally derived by a suitable asymptotic analysis of a membrane of finite thickness which is thin compared to the two outer domains it separates; it is a statement about concentration flux being proportional to the concentration difference across the membrane, accounting for chemical preference towards a particular phase. The boundary condition (3b) is a sublimit of (57) in the limit of $\Pi \rightarrow \infty$. If we were to use (57) instead of (3b), the full generality of (57) would appear in the distinguished limit of Case I using the current scalings of Case I (see Table 1) in addition to $\Pi=O\left(1 / \epsilon^{3}\right)$. This added generality does not affect matters significantly; it simply modifies (14a), the boundary condition 
we derive on the bacterial membrane to close the problem, to the following:

$$
\overline{\mathcal{K}} \bar{C}+\left(\bar{R}+\frac{1}{\epsilon^{3} \Pi}\right) \bar{D} \frac{\partial \bar{C}}{\partial \bar{r}}=\widehat{c}(\boldsymbol{x}, t) \quad \text { for } \bar{r}=\bar{R}(\boldsymbol{x}) .
$$

Replacing the scaling we make in section 4.5 with $\bar{r}=\bar{R} r, \bar{C}=\left(\epsilon^{3} \Pi \bar{R} \widehat{c} / \bar{D}(1+\right.$ $\left.\left.\epsilon^{3} \Pi \bar{R}\right)\right) w$, and $\bar{f}(\bar{C})=\epsilon^{3} \Pi \widehat{c} F(w) / \bar{R}\left(1+\epsilon^{3} \Pi \bar{R}\right)$, we recover the previous scaled equations (22) with $k=\epsilon^{3} \Pi \bar{R} \overline{\mathcal{K}} / \bar{D}\left(1+\epsilon^{3} \Pi \bar{R}\right)$. Under this new scaling the previous effective uptake (23) is replaced with

$$
\nu[\widehat{c}]=4 \pi \bar{R}^{2}\left(\left.\frac{\epsilon^{3} \Pi}{1+\epsilon^{3} \Pi \bar{R}} \frac{\partial w}{\partial r}\right|_{r=1}\right) \widehat{c} .
$$

For Case II, the full generality of (57) would appear using the current scalings of Case II (see Table 1) in addition to $\Pi=O(1 / \epsilon)$. In this case, the final upscaled equation (68) would still hold, but now with the boundary condition (64b) in the cell problem being replaced with

$$
\boldsymbol{n}_{\boldsymbol{y}} \cdot\left(\nabla_{\boldsymbol{y}} \xi_{i}-\boldsymbol{e}_{i}\right)=\frac{\epsilon \Pi}{\mathcal{K}}\left(\xi_{i}-\Xi_{i}\right) \quad \text { for } \boldsymbol{y} \in \partial \omega_{b}(\boldsymbol{x})
$$

Therefore, the results we derive in this paper can easily be modified to account for the more general coupling condition (57) instead of (3b).

We neglect any advection in this work, in order to focus on the distinguished limits that arise with diffusion and uptake as the dominant transport processes. Although this may appear to restrict our analysis to stagnant bacterial colonies, our results can actually be applied to a broader range of physical set-ups, including nonmotile microorganisms in the presence of a background flow. This is because nonmotile microorganisms will simply be advected with the flow, i.e., there is no relative movement between the flow and the microorganism motion, and in the frame of reference of the background flow, our analysis pertains as long as the lengthscale of fluid shear is significantly larger than the bacterial lengthscale.

In this paper, we investigate and quantify how the effective uptake and diffusivity depend on microscale bacterial properties. We show how a nonlinear pointwise uptake is transformed into an observable macroscale uptake with different nonlinear properties and how significantly different microscale behaviors can still result in the same observed uptake. Additionally, we show how the relative size of a nonunitary partition coefficient can affect in which of the observed uptake regimes one finds oneself. We emphasize that the reaction-diffusion system we consider is not restricted to bacteria; it can also be applied to many other single-celled microorganisms, such as cyanobacteria, microalgae, protozoa, and yeast. Moreover, the microscale framework we upscale in this paper is fairly general - diffusive transport past finite-volumetric sinks with a general nonlinear uptake. We hope that the framework and results we present here will be used and extended to other related problems in a wide range of physical applications.

Appendix A. Case II: The distinguished limit for the effective diffusivity. The distinguished limit for the effective diffusivity occurs when all dimensionless parameters in the system are of $O(1)$. While this limit is only a slight modification of the general classical case [12, section 5.3], now including an arbitrary partition coefficient across the internal boundary, it does provide the distinguished limit for 
the effective diffusivity. We therefore include the analysis for completeness. This case contains Case 1 from [9] as a sublimit.

To proceed, we introduce the asymptotic expansions

$$
c=c_{0}(\boldsymbol{x}, \boldsymbol{y}, t)+\epsilon c_{1}(\boldsymbol{x}, \boldsymbol{y}, t)+\epsilon^{2} c_{2}(\boldsymbol{x}, \boldsymbol{y}, t)+O\left(\epsilon^{3}\right)
$$

with the equivalent for $C$, substitute these into (8), and equate terms of equal magnitude.

At $O(1)$, we obtain solutions which are independent of $\boldsymbol{y}$, and thus $c_{0}=c_{0}(\boldsymbol{x}, t)$ and $C_{0}=C_{0}(\boldsymbol{x}, t)$ with $c_{0}=\mathcal{K} C_{0}$ and $c_{0}(\boldsymbol{x}, 0)=\mathcal{K} C_{0}(\boldsymbol{x}, 0)=c_{\text {init }}(\boldsymbol{x})$. To close the problem at leading-order, we must derive a solvability condition from higher asymptotic orders.

The $O(\epsilon)$ terms in (8) yield

$$
\begin{aligned}
0=\nabla_{\boldsymbol{y}}^{2} c_{1} \quad \text { for } \boldsymbol{y} \in \omega_{m}(\boldsymbol{x}), \quad 0=D \nabla_{\boldsymbol{y}}^{2} C_{1} & \text { for } \boldsymbol{y} \in \omega_{b}(\boldsymbol{x}), \\
c_{1}=\mathcal{K} C_{1}, \quad \boldsymbol{n}_{\boldsymbol{y}} \cdot\left(\nabla_{\boldsymbol{y}} c_{1}+\nabla_{\boldsymbol{x}} c_{0}\right)=D \boldsymbol{n}_{\boldsymbol{y}} \cdot\left(\nabla_{\boldsymbol{y}} C_{1}+\nabla_{\boldsymbol{x}} C_{0}\right) & \text { for } \boldsymbol{y} \in \partial \omega_{b}(\boldsymbol{x}), \\
c_{1} \text { periodic } & \text { for } \boldsymbol{y} \in \partial \omega .
\end{aligned}
$$

We can express solutions to (62) in the form

$$
\begin{aligned}
c_{1}(\boldsymbol{x}, \boldsymbol{y}, t) & =-\boldsymbol{\xi}(\boldsymbol{x}, \boldsymbol{y}) \cdot \nabla_{\boldsymbol{x}} c_{0}(\boldsymbol{x}, t)+\breve{c}_{1}(\boldsymbol{x}, t), \\
C_{1}(\boldsymbol{x}, \boldsymbol{y}, t) & =-\boldsymbol{\Xi}(\boldsymbol{x}, \boldsymbol{y}) \cdot \nabla_{\boldsymbol{x}} C_{0}(\boldsymbol{x}, t)+\breve{C}_{1}(\boldsymbol{x}, t),
\end{aligned}
$$

where $\breve{c}_{1}$ and $\breve{C}_{1}$ are functions of $\boldsymbol{x}$ and $t$ only that we do not need to calculate to obtain the leading-order homogenized problem. The components $\xi_{i}$ and $\Xi_{i}$ of the functions $\boldsymbol{\xi}$ and $\boldsymbol{\Xi}$ satisfy the cell problems

$$
\begin{aligned}
& 0=\nabla_{\boldsymbol{y}}^{2} \xi_{i} \quad \text { for } \boldsymbol{y} \in \omega_{m}(\boldsymbol{x}), \quad 0=D \nabla_{\boldsymbol{y}}^{2} \Xi_{i} \quad \text { for } \boldsymbol{y} \in \omega_{b}(\boldsymbol{x}), \\
& \xi_{i}=\Xi_{i} \text { for } \boldsymbol{y} \in \partial \omega_{b}(\boldsymbol{x}), \\
& \boldsymbol{n}_{\boldsymbol{y}} \cdot\left(\nabla_{\boldsymbol{y}} \xi_{i}-\frac{D}{\mathcal{K}} \nabla_{\boldsymbol{y}} \Xi_{i}\right)=\left(1-\frac{D}{\mathcal{K}}\right) \boldsymbol{n}_{\boldsymbol{y}} \cdot \boldsymbol{e}_{i} \quad \text { for } \boldsymbol{y} \in \partial \omega_{b}(\boldsymbol{x}), \\
& \xi_{i} \text { periodic for } \boldsymbol{y} \in \partial \omega \text {, }
\end{aligned}
$$

where $\boldsymbol{e}_{i}$ is the unit vector in the $y_{i}$-direction. The cell problem (64) depends on two parameters: the ratio $D / \mathcal{K}$, which appears explicitly in the cell problem, and $R$, the bacterial radius.

Finally, the $O\left(\epsilon^{2}\right)$ terms in (8) yield

$$
\begin{aligned}
& \frac{\partial C_{0}}{\partial t}=D \nabla_{\boldsymbol{y}} \cdot\left(\nabla_{\boldsymbol{y}} C_{2}+\nabla_{\boldsymbol{x}} C_{1}\right)+D \nabla_{\boldsymbol{x}} \cdot\left(\nabla_{\boldsymbol{y}} C_{1}+\nabla_{\boldsymbol{x}} C_{0}\right)-f\left(C_{0}\right) \quad \text { for } \boldsymbol{y} \in \omega_{b}(\boldsymbol{x}), \\
& c_{2}=\mathcal{K} C_{2} \text { for } \boldsymbol{y} \in \partial \omega_{b}(\boldsymbol{x}), \\
& \boldsymbol{n}_{\boldsymbol{y}} \cdot\left(\nabla_{\boldsymbol{y}} c_{2}+\nabla_{\boldsymbol{x}} c_{1}\right)-\nabla_{\boldsymbol{x}} R \cdot\left(\nabla_{\boldsymbol{y}} c_{1}+\nabla_{\boldsymbol{x}} c_{0}\right) \\
& =D\left(\boldsymbol{n}_{\boldsymbol{y}} \cdot\left(\nabla_{\boldsymbol{y}} C_{2}+\nabla_{\boldsymbol{x}} C_{1}\right)-\nabla_{\boldsymbol{x}} R \cdot\left(\nabla_{\boldsymbol{y}} C_{1}+\nabla_{\boldsymbol{x}} C_{0}\right)\right) \text { for } \boldsymbol{y} \in \partial \omega_{b}(\boldsymbol{x}) \text {, } \\
& c_{2} \text { periodic for } \boldsymbol{y} \in \partial \omega \text {. }
\end{aligned}
$$

Copyright $\odot$ by SIAM. Unauthorized reproduction of this article is prohibited. 
To derive effective equations for the averaged concentration (5), we integrate (65a) over $\omega_{m}(\boldsymbol{x})$ and $(65 \mathrm{~b})$ over the domain $\omega_{b}(\boldsymbol{x})$, sum the results, then apply the divergence theorem with the boundary conditions $(65 \mathrm{~d})-(65 \mathrm{e})$ to obtain

$$
\begin{gathered}
\int_{\omega_{m}(\boldsymbol{x})} \frac{\partial c_{0}}{\partial t} \mathrm{~d} \boldsymbol{y}+\int_{\omega_{b}(\boldsymbol{x})} \frac{\partial C_{0}}{\partial t} \mathrm{~d} \boldsymbol{y}=\int_{\omega_{m}(\boldsymbol{x})} \nabla_{\boldsymbol{x}} \cdot\left(\nabla_{\boldsymbol{y}} c_{1}+\nabla_{\boldsymbol{x}} c_{0}\right) \mathrm{d} \boldsymbol{y} \\
-\int_{\partial \omega_{b}(\boldsymbol{x})} \nabla_{\boldsymbol{x}} R \cdot\left(\nabla_{\boldsymbol{y}} c_{1}+\nabla_{\boldsymbol{x}} c_{0}\right) \mathrm{d} s+D \int_{\omega_{b}(\boldsymbol{x})} \nabla_{\boldsymbol{x}} \cdot\left(\nabla_{\boldsymbol{y}} C_{1}+\nabla_{\boldsymbol{x}} C_{0}\right) \mathrm{d} \boldsymbol{y} \\
-D \int_{\partial \omega_{b}(\boldsymbol{x})} \nabla_{\boldsymbol{x}} R \cdot\left(\nabla_{\boldsymbol{y}} C_{1}+\nabla_{\boldsymbol{x}} C_{0}\right) \mathrm{d} s-\int_{\omega_{b}(\boldsymbol{x})} f\left(C_{0}\right) \mathrm{d} \boldsymbol{y},
\end{gathered}
$$

where $\mathrm{d} s$ is the surface element of the bacterial membrane $\partial \omega_{b}(\boldsymbol{x})$. Using the Reynolds transport theorem to combine the first and second integrals on the right-hand side of (66) as well as the third and fourth integrals, we obtain

$$
\begin{aligned}
\left|\omega_{m}(\boldsymbol{x})\right| \frac{\partial c_{0}}{\partial t}+\left|\omega_{b}(\boldsymbol{x})\right| \frac{\partial C_{0}}{\partial t}= & \nabla_{\boldsymbol{x}} \cdot \int_{\omega_{m}(\boldsymbol{x})}\left(\nabla_{\boldsymbol{y}} c_{1}+\nabla_{\boldsymbol{x}} c_{0}\right) \mathrm{d} \boldsymbol{y} \\
& +D \nabla_{\boldsymbol{x}} \cdot \int_{\omega_{b}(\boldsymbol{x})}\left(\nabla_{\boldsymbol{y}} C_{1}+\nabla_{\boldsymbol{x}} C_{0}\right) \mathrm{d} \boldsymbol{y}-\left|\omega_{b}(\boldsymbol{x})\right| f\left(C_{0}\right) .
\end{aligned}
$$

We note that (67) could also have been obtained by summing the integrals of the full governing equations $(8 \mathrm{a})$ and $(8 \mathrm{~b})$ over their respective domains, using the coupling condition (8d), and applying the asymptotic ansatz (61).

To obtain a governing equation for the intrinsic averaged concentration $\widehat{c}$, we first note that $\widehat{c} \sim c_{0}$, which follows from the leading-order independence of $c_{0}$ on $\boldsymbol{y}$. We then use (63) to deduce that $\nabla_{\boldsymbol{y}} c_{1}=-\left(\mathbf{J}_{\boldsymbol{\xi}}^{\mathrm{T}}\right) \nabla_{\boldsymbol{x}} c_{0}$ and $\nabla_{\boldsymbol{y}} C_{1}=-\left(\mathbf{J}_{\mathfrak{\Xi}}^{\mathrm{T}}\right) \nabla_{\boldsymbol{x}} C_{0}$. Here, $\left(\mathbf{J}_{\xi}^{\mathrm{T}}\right)_{i j}=\partial \xi_{j} / \partial y_{i}$ and $\left(\mathbf{J}_{\Xi}^{\mathrm{T}}\right)_{i j}=\partial \Xi_{j} / \partial y_{i}$ are the transposes of the Jacobian matrices of $\boldsymbol{\xi}$ and $\boldsymbol{\Xi}$, respectively, these being the vector solutions to the cell problems defined in (64). Using these results, recalling that $c_{0}=\mathcal{K} C_{0}$ from the leading-order equations and that $\left|\omega_{m}\right|+\left|\omega_{b}\right|=1$, we can rearrange (67) to obtain a leading-order governing equation for $\widehat{c}$

$$
\frac{\partial \widehat{c}}{\partial t}=\frac{1}{\widehat{\alpha}(\boldsymbol{x})} \nabla_{\boldsymbol{x}} \cdot\left(\widehat{\mathbf{D}}(\boldsymbol{x}) \nabla_{\boldsymbol{x}} \widehat{c}\right)-\frac{\left|\omega_{b}(\boldsymbol{x})\right|}{\widehat{\alpha}(\boldsymbol{x})} f(\widehat{c} / \mathcal{K}), \quad \widehat{c}(\boldsymbol{x}, 0)=c_{\text {init }}(\boldsymbol{x}) .
$$

Here, we define

$$
\widehat{\alpha}(\boldsymbol{x})=1+\frac{1-\mathcal{K}}{\mathcal{K}}\left|\omega_{b}(\boldsymbol{x})\right|
$$

and the homogenized diffusion tensor

$$
\widehat{\mathbf{D}}(\boldsymbol{x})=\left(1+\frac{D-\mathcal{K}}{\mathcal{K}}\left|\omega_{b}\right|\right) \mathbf{I}-\int_{\omega_{m}(\boldsymbol{x})} \mathbf{J}_{\boldsymbol{\xi}}^{\mathrm{T}} \mathrm{d} \boldsymbol{y}-\frac{D}{\mathcal{K}} \int_{\omega_{b}(\boldsymbol{x})} \mathbf{J}_{\Xi}^{\mathrm{T}} \mathrm{d} \boldsymbol{y}
$$

where $\mathbf{I}$ is the three-dimensional identity matrix. Since we have spherical bacteria, the homogenized diffusion tensor is a multiple of the identity matrix due to the symmetry of the cell problem (64). That is,

$$
\int_{\omega_{m}(\boldsymbol{x})} \mathbf{J}_{\boldsymbol{\xi}}^{\mathrm{T}} \mathrm{d} \boldsymbol{y}=\left(\int_{\omega_{m}(\boldsymbol{x})} \partial \xi_{i} / \partial y_{i} \mathrm{~d} \boldsymbol{y}\right) \mathbf{I}, \quad \int_{\omega_{b}(\boldsymbol{x})} \mathbf{J}_{\Xi}^{\mathrm{T}} \mathrm{d} \boldsymbol{y}=\left(\int_{\omega_{b}(\boldsymbol{x})} \partial \Xi_{j} / \partial y_{j} \mathrm{~d} \boldsymbol{y}\right) \mathbf{I}
$$




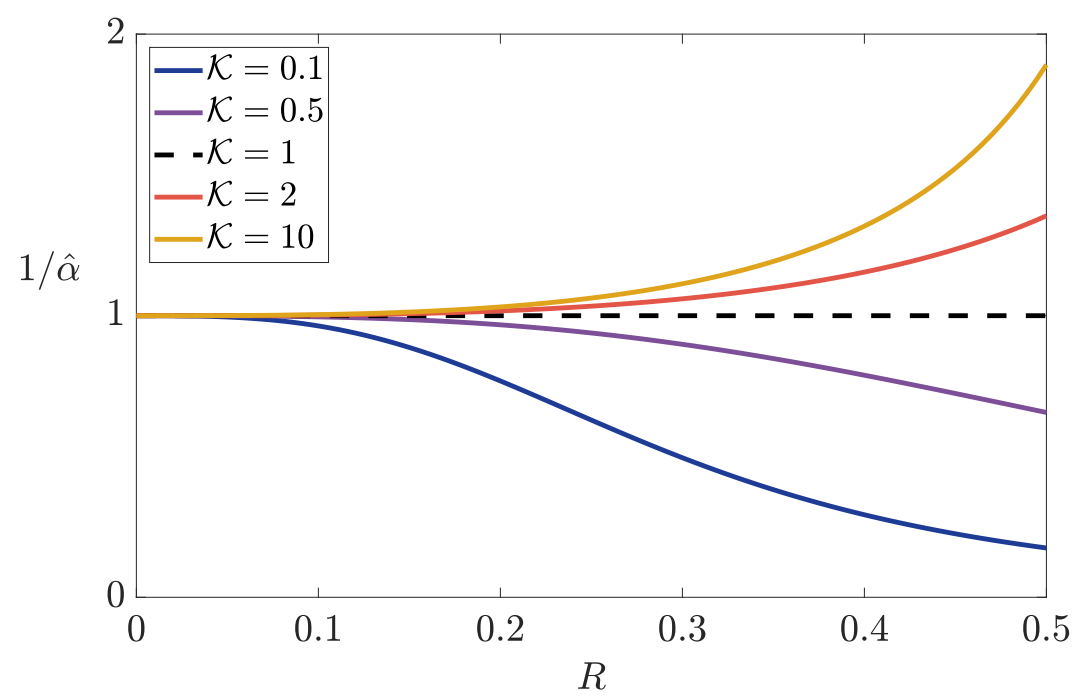

FIG. 7. The ratio of the observed effective uptake $\frac{\left|\omega_{b}\right|}{\widehat{\alpha}} f(\widehat{c} / \mathcal{K})$ to the total actual uptake within a bacterium $\left|\omega_{b}\right| f(\widehat{c} / \mathcal{K})$ varies in terms of $R$ and $\mathcal{K}$.

for $i, j=1,2,3$, with $\xi_{i}$ and $\Xi_{j}$ determined by (64). We emphasize that we do not invoke the summation convention in (69).

We have therefore derived an upscaled equation for the concentration evolution (68) in terms of the microscale system parameters. We see from (68a) that the effective uptake is equal to the product of the pointwise uptake, the bacterial volume, and a modifying factor of $1 / \widehat{\alpha}$. In comparison, in [9], where we consider a linear uptake and $\mathcal{K}=1$, the effective uptake in Case 1 is the product of the pointwise uptake and the bacterial volume with no modifying factor. As $\widehat{\alpha} \rightarrow 1$ when $\mathcal{K} \rightarrow 1$ from (68b), we are able to recover the limit of [9] in the current work. From the form of the effective uptake, we can see that introducing a nonlinearity does not affect the effective uptake significantly, as this nonlinearity is carried through the upscaling largely unscathed, simply accounting for the partition coefficient. However, the presence of the partition coefficient does modify the observed effective uptake in a nontrivial manner, due to the dependence of $\widehat{\alpha}$ on $\mathcal{K}$ (Figure 7). That is, as $1 / \widehat{\alpha}<1$ for $\mathcal{K}<1$ and vice versa, we may deduce that the observed uptake within a colony of bacteria will be smaller/larger than naively assuming that the effective uptake is the product of bacterial volume and pointwise uptake if the partition coefficient $\mathcal{K}$ is smaller/greater than 1 , i.e., if the concentration preference is for the cytoplasm/ECM. The reason for this discrepancy in Case 1 is because the chemical concentration will be observed in terms of the intrinsicaveraged concentration, whereas the measured uptake will be in terms of the moles of chemical species removed, a quantity captured by the volumetric-averaged concentration. Indeed, this discrepancy would vanish if one considered the volumetric-averaged concentration version instead of the intrinsic-averaged concentration version (68).

The homogenized diffusion tensor defined in (68c) will represent the most general effective diffusivity in the system. Moreover, (68c) represents a more general effective diffusivity than that derived in [9]. From the functional form of (68c), we see that while introducing a nonunitary partition coefficient does have a significant effect on the effective diffusivity, introducing a nonlinear uptake does not have any leadingorder effect. 
Appendix B. Steady state stability analysis. We consider a small timedependent perturbation away from a steady-state solution $\bar{C}^{*}(\bar{r})$ of $(21)$

$$
\bar{C}(\bar{r}, t)=\bar{C}^{*}(\bar{r})+e^{\lambda t} \Theta(\bar{r}),
$$

where $\Theta \ll \bar{C}^{*}$. Here, $\Theta$ satisfies the eigenvalue problem

$$
0=\frac{\bar{D}}{\bar{r}^{2}} \frac{\partial}{\partial \bar{r}}\left(\bar{r}^{2} \frac{\partial \Theta}{\partial \bar{r}}\right)-\left(\bar{f}^{\prime}\left(\bar{C}^{*}\right)+\lambda\right) \Theta \quad \text { for } \bar{r}<\bar{R}(\boldsymbol{x})
$$

for the eigenvalue $\lambda$, with boundary conditions

$$
\frac{\partial \Theta}{\partial \bar{r}}=0 \quad \text { for } \bar{r}=0, \quad \overline{\mathcal{K}} \Theta+\bar{R} \bar{D} \frac{\partial \Theta}{\partial \bar{r}}=0 \quad \text { for } \bar{r}=\bar{R} .
$$

Multiplying (71a) by $\bar{r}^{2} \Theta$ and integrating over $\bar{r} \in(0, \bar{R})$ to form the Rayleigh quotient, we obtain

$$
\lambda=-\frac{\bar{R} \overline{\mathcal{K}} \Theta^{2}(\bar{R})+\int_{0}^{\bar{R}} \bar{r}^{2}\left(\bar{D}\left(\Theta^{\prime}\right)^{2}+\bar{f}^{\prime}\left(\bar{C}^{*}\right) \Theta^{2}\right) \mathrm{d} \bar{r}}{\int_{0}^{\bar{R}} \bar{r}^{2} \Theta^{2} \mathrm{~d} \bar{r}},
$$

where we used (71b) to simplify the term outside the integrals. From (72), we may immediately deduce that if $\bar{f}^{\prime}\left(\bar{C}^{*}\right) \geqslant 0$ everywhere within the domain we have $\lambda \leqslant 0$, and hence any steady state is stable if $\bar{f}^{\prime}\left(\bar{C}^{*}\right) \geqslant 0$. That is, any steady state is stable if the uptake $\bar{f}(\bar{C})$ is monotonically nondecreasing. An interesting corollary of this is that it may be possible to have unstable steady states for this problem if there are strong inhibition effects as the concentration increases.

\section{REFERENCES}

[1] T. Arbogast, J. Douglas, JR., And U. Hornung, Derivation of the double porosity model of single phase flow via homogenization theory, SIAM J. Math. Anal., 21 (1990), pp. 823-836.

[2] A. Bensoussan, J.-L. Lions, and G. Papanicolaou, Asymptotic Analysis for Periodic Structures, North-Holland, Amsterdam, 1978.

[3] M. Bruna And S. J. Chapman, Diffusion in spatially varying porous media, SIAM J. Appl. Math., 75 (2015), pp. 1648-1674.

[4] I. L. Chernyavsky, I. L. Dryden, And O. E. Jensen, Characterizing the multiscale structure of fluctuations of transported quantities in a disordered medium, IMA J Appl Math, 77 (2012), pp. 697-725.

[5] I. L. Chernyavsky, L. Leach, I. L. Dryden, and O. E. Jensen, Transport in the placenta: Homogenizing haemodynamics in a disordered medium, Philos. Trans. A, 369 (2011), pp. $4162-4182$.

[6] D. Cioranescu and F. Murat, A strange term coming from nowhere, in Topics in the Mathematical Modelling of Composite Materials, Springer, Berlin, 1997, pp. 45-93.

[7] M. P. Dalwadi, M. Bruna, and I. M. Griffiths, A multiscale method to calculate filter blockage, J. Fluid Mech., 809 (2016), pp. 264-289.

[8] M. P. Dalwadi, I. M. GRiffiths, and M. Bruna, Understanding how porosity gradients can make a better filter using homogenization theory, Proc. A, 471 (2015).

[9] M. P. Dalwadi, Y. Wang, J. R. King, and N. P. Minton, Upscaling diffusion through firstorder volumetric sinks: A homogenization of bacterial nutrient uptake, SIAM J. Appl. Math., 78 (2018), pp. 1300-1329.

[10] D. Davies, Understanding biofilm resistance to antibacterial agents, Nat. Rev. Drug Discov., 2 (2003), pp. 114-122.

[11] L. Hall-Stoodley, J. W. Costerton, and P. Stoodley, Bacterial biofilms: From the natural environment to infectious diseases, Nat. Rev. Microbiol., 2 (2004), pp. 95-108.

[12] M. H. Holmes, Introduction to Perturbation Methods, Texts Appl. Math. 20, Springer, Berlin, 2012.

Copyright $@$ by SIAM. Unauthorized reproduction of this article is prohibited. 
[13] U. Hornung, Homogenization and Porous Media, Interdiscip. Appl. Math. 6, Springer, Berlin, 2012.

[14] U. Hornung, W. JÄGer, And A. Mikelić, Reactive transport through an array of cells with semi-permeable membranes, Math. Model. Numer. Anal., 28 (1994), pp. 59-94.

[15] A. Leo, C. Hansch, and D. Elkins, Partition coefficients and their uses, Chem. Rev., 71 (1971), pp. 525-616.

[16] P. A. Levin And E. R. Angert, Small but mighty: Cell size and bacteria, Cold Spring Harbor Perspec. Biol., 7 (2015), a019216.

[17] H. Märkl, C. Zenneck, A. C. H. Dubach, and J. C. Ogbonna, Cultivation of Escherichia coli to high cell densities in a dialysis reactor, Appl. Microbiol. Biotech., 39 (1993), pp. 4852 .

[18] F. Murat and D. Cioranescu, Un terme étrange venu d'ailleurs, in Non-Linear Partial Differential Equations and Their Applications, Volumes II and III, Collége de France Seminar, 1982, pp. 98-138.

[19] J. D. MurRay, Mathematical Biology: I. An Introduction, Interdiscip. Appl. Math. 17, Springer, Berlin, 2007.

[20] R. D. O’Dea, M. R. Nelson, A. J. El Haj, S. L. Waters, and H. M. Byrne, A multiscale analysis of nutrient transport and biological tissue growth in vitro, Math. Med. Biol., 32 (2015), pp. 345-366.

[21] R. Penta, D. Ambrosi, and A. Quarteroni, Multiscale homogenization for fluid and drug transport in vascularized malignant tissues, Math. Models Methods Appl. Sci., 25 (2015), pp. $79-108$.

[22] M. Ptashnyk, T. Roose, And G. J. D. Kirk, Diffusion of strongly sorbed solutes in soil: A dual-porosity model allowing for slow access to sorption sites and time-dependent sorption reactions, Eur. J. Soil Sci., 61 (2010), pp. 108-119.

[23] G. Richardson and S. J. Chapman, Derivation of the bidomain equations for a beating heart with a general microstructure, SIAM J. Appl. Math., 71 (2011), pp. 657-675.

[24] M. J. Russell And O. E. Jensen, Homogenization approximations for unidirectional transport past randomly distributed sinks, IMA J. Appl. Math., 85 (2020), pp. 161-189.

[25] M. J. Russell, O. E. Jensen, And T. Galla, Stochastic transport in the presence of spatial disorder: Fluctuation-induced corrections to homogenization, Phys. Rev. E, 94 (2016), 042121.

[26] E. SÁnChez-PALEncia, Non-Homogeneous Media and Vibration Theory, Lecture Notes in Phys. 127, Springer, Berlin, 1980.

[27] R. J. Shipley and S. J. Chapman, Multiscale modelling of fluid and drug transport in vascular tumours, Bull. Math. Biol., 72 (2010), pp. 1464-1491.

[28] P. S. Stewart And J. W. Costerton, Antibiotic resistance of bacteria in biofilms, Lancet, 358 (2001), pp. 135-138.

[29] M. van Dyke, Perturbation Methods in Fluid Dynamics, Parabolic Press, Stanford, CA, 1975.

[30] T. L. VAN NoORden, Crystal precipitation and dissolution in a porous medium: Effective equations and numerical experiments, Multiscale Model. Simul., 7 (2009), pp. 1220-1236.

[31] T. L. Van Noorden And A. Muntean, Homogenisation of a locally periodic medium with areas of low and high diffusivity, European J. Appl. Math., 22 (2011), pp. 493-516.

Copyright $@$ by SIAM. Unauthorized reproduction of this article is prohibited. 